\title{
Identification of an Oligosaccharide Dehydrogenase from Pycnoporus Cinnabarinus Provides Insights into Fungal Breakdown of Lignocellulose
}

\section{Gabriele Cerutti}

Sapienza University of Rome: Universita degli Studi di Roma La Sapienza

\section{Elena Gugole}

Sapienza University of Rome: Universita degli Studi di Roma La Sapienza

\section{Linda Celeste Montemiglio}

Istituto di Biologia e Patologia Molecolari Consiglio Nazionale delle Ricerche

\section{Annick Turbé-Doan}

INRAE: Institut National de Recherche pour l'Agriculture l'Alimentation et l'Environnement

\section{Dehbia Chena}

INRAE: Institut National de Recherche pour l'Agriculture I'Alimentation et l'Environnement

\section{David Navarro}

INRAE: Institut National de Recherche pour l'Agriculture l'Alimentation et l'Environnement

Anne Lomascolo

AMU: Aix-Marseille Universite

\section{François Piumi}

École Nationale Vétérinaire d'Alfort: Ecole Nationale Veterinaire d'Alfort

\section{Cécile Exertier}

Sapienza University of Rome: Universita degli Studi di Roma La Sapienza

\section{Ida Freda}

Sapienza University of Rome: Universita degli Studi di Roma La Sapienza

\section{Beatrice Vallone}

Sapienza University of Rome: Universita degli Studi di Roma La Sapienza

\section{Eric Record}

INRAE: Institut National de Recherche pour l'Agriculture l'Alimentation et l'Environnement

\section{Carmelinda Savino}

Istituto di Biologia e Patologia Molecolari Consiglio Nazionale delle Ricerche

giuliano sciara ( $\nabla$ giuliano.sciara@inrae.fr)

INRAe UMR1163 Biodiversité et Biotechnologie Fongiques https://orcid.org/0000-0002-3790-747X 
Keywords: Oligosaccharide dehydrogenase, Redox enzymes, Pycnoporus cinnabarinus, X-ray crystallography, Lignocellulose degradation.

Posted Date: February 19th, 2021

DOI: https://doi.org/10.21203/rs.3.rs-237113/v1

License: (c) (1) This work is licensed under a Creative Commons Attribution 4.0 International License. Read Full License

Version of Record: A version of this preprint was published at Biotechnology for Biofuels on July 22nd, 2021. See the published version at https://doi.org/10.1186/s13068-021-02003-y. 


\section{Identification of an oligosaccharide dehydrogenase from Pycnoporus cinnabarinus provides insights into fungal breakdown of lignocellulose}

Gabriele Cerutti ${ }^{1,2 \dagger}$, Elena Gugole ${ }^{1}$, Linda Celeste Montemiglio ${ }^{3}$, Annick Turbé-Doan ${ }^{5}$, Dehbia Chena $^{5}$, David Navarro ${ }^{5}$, Anne Lomascolo ${ }^{5}$, François Piumi ${ }^{4,5}$, Cécile Exertier ${ }^{1}$, Ida Freda ${ }^{1}$, Beatrice Vallone $e^{1,2,3}$, Eric Record ${ }^{5}$, Carmelinda Savino ${ }^{3 *}$ and Giuliano Sciara ${ }^{5 *}$.

${ }^{1}$ Dipartimento di Scienze Biochimiche “A. Rossi Fanelli”, Sapienza University of Rome, Rome, Italy. ${ }^{2}$ Istituto Pasteur-Fondazione Cenci Bolognetti, Sapienza University of Rome, Rome, Italy.

${ }^{3}$ Istituto di Biologia e Patologia Molecolari, National Research Council, Rome, Italy.

${ }^{4}$ Anses, INRAE, Ecole Nationale Vétérinaire d'Alfort, Université Paris-Est, UMR1161 Virologie, Maisons-Alfort, France.

${ }^{5}$ Aix-Marseille Université, INRAE, UMR1163 Biodiversité et Biotechnologie Fongiques, Marseille, France.

'Present address: Zuckerman Mind Brain Behavior Institute, Columbia University, New York, NY 10029, USA

*Corresponding authors: To whom correspondence should be addressed: Carmelinda Savino, Consiglio Nazionale delle Ricerche (CNR) Institute of Molecular Biology and Pathology, P.le A. Moro 5, 00185 Rome, Italy. E-mail: carmelinda.savino@cnr.it; Giuliano Sciara, INRAE, UMR1163 Biodiversité et Biotechnologie Fongiques, BBF UMR1163 INRAe / Aix-Marseille Université, 163 Avenue de Luminy, 13009 Marseille, France. E-mail: giuliano.sciara@inrae.fr 


\section{Abstract}

Background: Fungal glucose dehydrogenases (GDHs) are FAD-dependent enzymes belonging to the glucose-methanol-choline oxidoreductase superfamily. These enzymes are classified in the "Auxiliary Activity" family 3 (AA3) of the Carbohydrate-Active enZymes database, and more specifically in subfamily AA3_2, that also includes the closely related flavoenzymes aryl-alcohol oxidase and glucose 1-oxidase. Based on sequence similarity to known fungal GDHs, an AA3_2 enzyme active on glucose was identified in the genome of Pycnoporus cinnabarinus, a model Basidiomycete able to completely degrade lignin.

Results: In our work, substrate screening and functional characterization showed an unexpected preferential activity of this enzyme toward oligosaccharides containing a $\beta(1 \rightarrow 3)$ glycosidic bond, with the highest efficiency observed for the disaccharide laminaribiose. Despite its sequence similarity to GDHs, we defined a novel enzymatic activity, namely oligosaccharide dehydrogenase $(\mathrm{ODH})$, for this enzyme. The crystallographic structures of ODH in the sugar-free form and in complex with glucose and laminaribiose unveiled a peculiar saccharide recognition mechanism which is not shared with previously characterized AA3 oxidoreductases and accounts for ODH preferential activity toward oligosaccharides. The sugar molecules in the active site of ODH are mainly stabilized through $\mathrm{CH}-\pi$ interactions with aromatic residues rather than through hydrogen bonds with highly conserved residues, as observed instead for the fungal glucose dehydrogenases and oxidases characterized to date. Finally, three sugar-binding sites were identified on ODH external surface, which were not previously observed and might be of importance in the physiological scenario.

Conclusions: Structure-function analysis of ODH is consistent with its role as an auxiliary enzyme in lignocellulose degradation and unveils yet another enzymatic function within the AA3 family of the Carbohydrate-Active enZymes database. Our findings allow deciphering the molecular determinants of substrate binding and provide insight into the physiological role of ODH, opening new perspectives to exploit biodiversity for lignocellulose transformation into fuels and chemicals. 
Keywords: Oligosaccharide dehydrogenase, Redox enzymes, Pycnoporus cinnabarinus, X-ray crystallography, Lignocellulose degradation-

\section{Background}

The woody material of plants is a complex mixture of carbon-based polymers, mainly cellulose, hemicellulose and lignin, collectively called lignocellulosic biomass or lignocellulose. Cellulose is the most abundant biopolymer on earth and lignin, a heterogenous polymer assembled from differently methoxylated aromatic alcohols, accounts for about $25 \%$ of removable organic matter in the biosphere [1]. White-rot fungi are saprotrophic organisms able to effectively and selectively degrade lignocellulose. This is achieved through a wide arsenal of enzymes secreted by the fungus, which act in synergy to perform lignin enzymatic combustion [2]. Among them, some redox enzymes are classified within the "Auxiliary Activities" (AA) group [3] of the Carbohydrate-Active enZymes (CAZy) database, a curated collection of enzymes involved in carbohydrate transformations and lignocellulolysis [4]. Pycnoporus cinnabarinus (syn. Trametes cinnabarina) is a white-rot fungus known for its very efficient lignocellulose degrading properties, whose genome encodes for a large enzymatic arsenal of CAZymes, including lignin degrading enzymes: 5 laccases (CAZy family AA1), 9 class-II peroxidases (AA2) and 24 flavoenzymes (AA3). Among the latter, 19 belong to the glucose/aryl-alcohol oxidase/dehydrogenase group (subfamily AA3_2) [5]. This enzymatic versatility allowed $P$. cinnabarinus to stand out as a microorganism of choice for the biotransformation of aromatic compounds deriving from raw plant materials, with the aim of producing high-value products such as pharmaceuticals, antioxidants and aromas [6-9]. The use of lignin as a natural source of chemicals and biofuels represents an extremely promising target in the context of green chemistry and biorefinery, since it is currently regarded as one of the causes of lignocellulose recalcitrance to industrial treatments and as a low-grade by-product of industrial activities that employ cellulose and hemicellulose [10]. In this context, a detailed characterization of 
the biochemical machinery underlying lignocellulose and lignin degradation by white-rot fungi, like P. cinnabarinus, is required to develop novel biotechnologies for lignin valorization.

The first step of fungal lignin degradation in vivo is laccase-mediated oxidative attack, responsible for the formation of unstable radical species known as phenoxy radicals [11]; while in vitro these laccase-generated radicals lead to lignin re-polymerization, some unknown physiological mechanism enables fungi to completely degrade lignin. AA3 enzymes have been proposed to play a role in reducing and therefore deactivating phenoxy radicals, by oxidizing lignin and polysaccharide degradation products [12-14].

The members of the AA3 CAZy family are FAD-dependent enzymes that belong to the glucosemethanol-choline (GMC) oxidoreductase superfamily [15, 16]. Four AA3 subfamilies have been described that account for different FAD binding modes, enzymatic mechanisms and substrate preferences [17]. Subfamily AA3_2 includes eight phylogenetically distant clades of genes, coding for enzymes of unknown function (six clades), for aryl-alcohol oxidases (EC 1.1.3.7) and dehydrogenases (1 clade), as well as for glucose oxidases (GOXs; EC 1.1.3.4) and glucose dehydrogenases (GDHs; EC 1.1.5.9) [13]. Within the latter GOX/GDH clade, finally, phylogenetic analysis suggests the existence of one group of well characterized GOXs, and three groups of GDHs $[16,17]$ : GDH class-I (Ascomycota), including most of the already characterized GDHs; GDH classII (Ascomycota), phylogenetically related to class-I and including enzymes not yet characterized; and GDH class-III, including mostly proteins from Basidiomycota, but also two phylogenetically related groups of proteins from each phylum respectively, also reminiscent in sequence to GOXs [16]. In this work we study the only enzyme characterized within the GDH class-III subclade, that we previously shown to be active on D-glucose (GLC) [14].

The catalytic cycle of AA3_2 enzymes is thought to consist of a hydride and proton transfer from an oxidizable substrate to a final electron and proton acceptor [18-22], and it can be divided into two half-reactions. In the case of GDH and GOX, during the first half-reaction, deprotonation of GLC O1 hydroxyl triggers the transfer of two electrons and two protons to the oxidized FAD cofactor in its 
resting state. Once reduced, $\mathrm{FADH}_{2}$ is able to reverse the transfer to either oxygen (GOXs) or to a variety of aromatic electron acceptors like quinones (GDHs) [23], as shown in Figure 1, and possibly to phenoxy radicals as mentioned above. Within the physiological scenario, the latter reaction has been proposed to inhibit lignin repolymerization in vivo $[12,13]$, however other biological roles have been proposed for AA3 dehydrogenases, such as providing reduced hydroquinones for lytic polysaccharide monooxygenase (LPMO) catalysis. Finally, oxidases would generate hydrogen peroxide for lytic polysaccharide monooxygenases and peroxidases [17].

In this study we report the structural and functional characterization of a AA3_2 flavoenzyme from the white-rot fungus $P$. cinnabarinus and we give the evidence for a novel enzymatic activity within the GOX/GDH clade of fungal oxidoreductases. This flavoenzyme (GenBank: CDO69819.1; UniProtKB/TrEMBL: A0A060SC37) was previously assigned as a GDH class-III for its measurable activity on GLC [14], but here we show that it acts more efficiently as an oligosaccharide dehydrogenase (ODH). We used spectrophotometric methods to evaluate substrate specificity and kinetic parameters toward a selected set of sugar substrates, observing that GLC is not the preferred substrate. ODH showed instead a marked preference for oligosaccharides in which the reducing glucosyl unit is linked to the adjacent glucose by a $\beta(1 \rightarrow 3)$ glycosidic bond, as in the disaccharide laminaribiose (LMB), which we identified as the best substrate within selected set. We determined the crystallographic structure of ODH in the ligand-free form and in complex with GLC and LMB. Structural comparison between native and sugar-bound ODH reveals a substrate-binding mechanism which is not shared with any GDH and GOX characterized so far and which accounts for its preferred activity toward $\beta(1 \rightarrow 3)$-containing oligosaccharides. Finally, structure-function analysis of ODH raises new questions about phylogeny and functions of fungal AA3 enzymes, providing insight into fungal lignocellulose degradation and contributing valuable information for future developments of lignin biorefinery.

\section{Results}




\section{Evaluation of substrate specificity and kinetic analysis of ODH}

To gain insight into the biological function of ODH, a set of fourteen sugars, differing in their carbon atom number and stereochemistry, oligomeric state and glycosidic bonds (see Additional file 1, Fig. S1), were tested by following DCIP reduction due to ODH enzymatic activity (Fig. 2). Among the inquired monosaccharides, ODH displays the highest activity toward GLC, as previously reported [14].

The screening of disaccharides revealed that ODH discriminates between different types of glycosidic linkage. More in detail, only negligible or no activity was observed if the reducing glucosyl unit was linked to the adjacent monosaccharide by a $\beta(1 \rightarrow 4)$ glycosidic bond, as in lactose (LAC) and cellobiose (CLB), or by a $\alpha(1 \rightarrow 4)$ linkage, as present in maltose (MAL). Conversely, a pronounced enzymatic activity was detected for the $\beta(1 \rightarrow 3)$ disaccharide LMB, which behaved as the best substrate and lead to complete reduction of DCIP after $3 \mathrm{~h}$, only comparable to what observed using 100 times higher GLC concentration.

The analysis of trisaccharide oxidation provides further insight into substrate specificity, confirming that $\mathrm{ODH}$ has a preference toward sugars containing $\beta(1 \rightarrow 3)$-linked reducing glucose, with no activity observed toward cellotriose (CLT) and 1,3;1,4 $\beta$-glucotriose A (GTA), containing a $\beta(1 \rightarrow 4)$ bound reducing sugar unit. Moreover, ODH is active only if the second glycosidic bond starting from the trisaccharide reducing end is $\beta(1 \rightarrow 4)$ and not $\beta(1 \rightarrow 3)$, as in 1,$3 ; 1,4 \beta$-glucotriose $B(\mathrm{GTB})$ and laminaritriose (LMT) respectively. As a further confirmation of these preferences, we detected no or only negligible activity testing tetrasaccharides containing all $\beta(1 \rightarrow 3)$ and all $\beta(1 \rightarrow 4)$ linkages, namely laminaritetraose (LMTT) and cellotetraose (CLTT). In order to confirm the chemical nature of the enzymatic reaction products, LC-MS experiments were carried out, showing that GLC was converted by ODH to gluconolactone (not shown), as previously reported [14], and LMB to laminaribionolactone, by oxidation of glucose $\mathrm{C} 1$ hydroxyl (see Additional file 1, Fig. S2). 
A more extensive enzymatic characterization was carried out using $\mathrm{LMB}$, the best substrate in comparison to GLC. Unfortunately, it was impossible to measure the maximal rate of the reaction for both substrates. Indeed even though the enzymatic kinetics performed with GLC seems to follow a simple Michaelis-Menten model up to 1.5 M GLC (Fig. 3), however, at higher concentrations it displays a deviation from the hyperbola equation, as the measured initial rate decreased with increasing GLC concentrations (see Additional file 1, Fig. S3). Regarding LMB, completion of the kinetic curve was prevented by its solubility limit of the substrate $(150 \mathrm{mM})$, and we did not achieved enzyme saturation with the highest measurable concentration of LMB (115.5 mM). Therefore, apparent steady-state kinetic parameters (Table 1) were obtained from fitting the standard MichaelisMenten equation up to $1.5 \mathrm{M}$ GLC and $115.5 \mathrm{mM}$ LMB (Fig. 3).

Table 1 Apparent ODH kinetic constants for GLC and LMB.

\begin{tabular}{lcccc}
\hline & $\mathbf{K}_{\mathbf{M}}(\mathrm{mM})$ & $\boldsymbol{k}_{\text {cat }}\left(\mathrm{s}^{-1}\right)$ & $\boldsymbol{k}_{\text {cat }} / \mathbf{K}_{\mathbf{M}} *\left(\mathrm{M}^{-1} \mathrm{~s}^{-1}\right)$ & $\boldsymbol{k}_{\text {cat }} / \mathbf{K}_{\mathbf{M}} * *\left(\mathrm{M}^{-1} \mathrm{~s}^{-1}\right)$ \\
\hline & & & & \\
GLC & $755 \pm 110$ & $50 \pm 3$ & $67 \pm 10$ & $47 \pm 1$ \\
LMB & $77 \pm 10$ & $71 \pm 4$ & $917 \pm 129$ & $777 \pm 21$
\end{tabular}

* $k_{\text {cat }} / \mathrm{K}_{\mathrm{M}}$ calculated from the apparent $\mathrm{K}_{\mathrm{M}}$ and $k_{\text {cat }}$ estimated from fitting the Michaelis-Menten hyperbola to data points (Fig. 3).

** $k_{\text {cat }} / \mathrm{K}_{\mathrm{M}}$ calculated as the slope of the tangent of the Michaelis-Menten hyperbola in its linear region (insets in Fig. 3).

The apparent $\mathrm{K}_{\mathrm{M}}$ estimated for GLC is comparable to what reported previously [14]. Comparative analysis of the resulting apparent kinetic parameters indicates ten-fold higher $\mathrm{K}_{\mathrm{M}}$ values toward GLC with respect to LMB, but comparable maximum turnover rates $\left(k_{c a t}\right)$ (Table 1$)$. As a result, LMB derived specificity constant $\left(k_{c a t} / \mathrm{K}_{\mathrm{M}}\right)$ of the disaccharide exceeds more than 13 times the one estimated for GLC. To support this observation, we estimated the relative specificity of ODH for 
GLC and LMB was estimated by measuring the initial rates in the linear region of the MichaelisMenten plot, in which the substrate concentration is significantly smaller than the measured $\mathrm{K}_{M}$ values. In these conditions, the slope of the tangent of the Michaelis-Menten hyperbola at its origin corresponds to $k_{\text {cat }} / \mathrm{K}_{\mathrm{M}}$, and it is derived experimentally (Fig. 3, insets). The specificity constants determined by both approaches are consistent (Table 1) and confirm the higher ODH specificity toward LMB. The apparent values of the kinetic parameters suggest that the consumption rate of the two substrates is very similar, and that enhanced catalytic efficiency is due to preferential binding of LMB. A simple substrate inhibition model did not allow to satisfactorily fit the initial velocity data including high GLC (up to $3 \mathrm{M}$ ) points (see Additional file 1, Fig. S3). At these extreme GLC concentrations, changes in refractive index and viscosity may also affect the DCIP extinction coefficient [24], whereas increased viscosity may negatively affect catalytic efficiency, due to higher molecular friction in solution and decreased enzyme conformational freedom [25].

\section{Structural features of ligand-free ODH}

$\mathrm{ODH}$ crystals belong to $\mathrm{P} 2{ }_{1} 2_{1} 2_{1}$ space group and contain one protein molecule per asymmetric unit; as such, no 2-fold axis nor non-crystallographic symmetries are observed. This is consistent with ODH being a monomer, as previously observed for GDH from Aspergillus flavus (AfGDH) [20], whereas GOXs from Ascomycota are known to be dimeric [18, 19]. ODH crystals are bright yellow, suggesting that the FAD cofactor is in the oxidized resting state. Data collection and refinement statistics of all structures are summarized in Table 2. The final structure of ligand-free ODH (PDB entry 6XUT). That was refined up to $1.6 \AA$ resolution, is shown in Fig. 4, together with secondary structure assignment and with structural alignment to AfGDH (PDB: 4YNT) and GOX from Aspergillus niger (AnGOX; PDB: 1CF3). Both AfGDH and AnGOX are closely related to ODH and represent the reference structures of AA3_2 enzymes from the GOX/GDH clade. Structure-based sequence alignment of ODH with the two enzymes (Fig. 4) indicates that $A f G D H$ and $A n G O X$ share respectively, $36.4 \%$ and $34.3 \%$ identity with $\mathrm{ODH}$, as well as $1.2 \AA$ and $1.3 \AA$ average r.m.s.d. of C $\alpha$ 
atom positions. Conservation of key ODH residues throughout the 7 subclades of the GOX/GDH AA3_2 enzymes is reported in Additional file 1 (Fig. S4). The overall structure of ODH consists of $20 \alpha$-helices and $18 \beta$-strands organized in two domains, the FAD-binding domain and the substratebinding domain (Fig. 4), as in all GMC oxidoreductases [18-20]. The $\beta$-strands are arranged in $5 \beta$ sheets named following the nomenclature introduced for AnGOX [18].

Table 2 X-ray diffraction data collection and structure refinement statistics. Values in parentheses are for the highest-resolution shell.

\begin{tabular}{|c|c|c|c|}
\hline & $\begin{array}{l}\text { Ligand-free ODH } \\
\text { 6XUT } \\
\end{array}$ & $\begin{array}{l}\text { ODH-GLC } \\
\text { 6XUU }\end{array}$ & $\begin{array}{l}\text { ODH-LMB } \\
\text { 6XUV } \\
\end{array}$ \\
\hline \multicolumn{4}{|l|}{ Data collection } \\
\hline Space group & $\mathrm{P} 2{ }_{1}{ }_{1} 2_{1}$ & & \\
\hline Unit-cell dimensions $(\AA)$ & $\begin{array}{l}a=48.87 \\
b=61.59 \\
c=195.09\end{array}$ & & \\
\hline Resolution range $(\AA)$ & $\begin{array}{l}97.55-1.43 \\
(1.57-1.43)\end{array}$ & $\begin{array}{l}49.04-1.57 \\
(1.67-1.57)\end{array}$ & $\begin{array}{l}97.33-1.75 \\
(2.02-1.75)\end{array}$ \\
\hline Number of observations & $1391283(295026)$ & $1064709(190424)$ & 770177 (268996) \\
\hline Unique reflections & $128942(25836)$ & $103996(29033)$ & $59350(20470)$ \\
\hline Completeness $(\%)$ & $99.9(99.7)$ & $99.6(98.8)$ & $99.9(99.8)$ \\
\hline Redundancy & $12.7(11.4)$ & $6.6(6.6)$ & $13.0(13.1)$ \\
\hline $\mathrm{I} / \sigma(\mathrm{I})$ & $14.5(1.72)$ & $12.0(1.36)$ & $7.0(1.97)$ \\
\hline $\mathrm{R}_{\text {merge }}{ }^{\mathrm{a}}(\%)$ & $7.1(139.0)$ & $9.0(141.0)$ & $17.5(156.1)$ \\
\hline $\mathrm{CC}_{1 / 2}$ & $100(79.5)$ & $99.9(55.8)$ & $99.9(80.8)$ \\
\hline Wilson B-value $\left(\AA^{2}\right)$ & 22.0 & 28.6 & 25.9 \\
\hline \multicolumn{4}{|l|}{ Refinement } \\
\hline Resolution range $(\AA)$ & $97.55-1.60$ & $49.04-1.57$ & $97.33-1.75$ \\
\hline $\begin{array}{l}\text { Protein molecules per } \\
\text { asymmetric unit } \\
\mathrm{R}_{\text {work }} / R_{\text {free }}{ }^{b}\end{array}$ & $\begin{array}{l}1 \\
0.165 / 0.196\end{array}$ & $\begin{array}{l}1 \\
0.162 / 0.189\end{array}$ & $\begin{array}{l}1 \\
0.168 / 0.207\end{array}$ \\
\hline \multicolumn{4}{|l|}{$\begin{array}{l}\text { Deviations from ideal } \\
\text { geometry }\end{array}$} \\
\hline Bond $(\AA)$ & 0.0132 & 0.0127 & 0.0138 \\
\hline Angles $(\AA)$ & 1.713 & 1.856 & 2.005 \\
\hline $\begin{array}{l}\text { Ramachandran plot }(\%) \\
\text { Favored/allowed/outliers }\end{array}$ & $95.4 / 4.6 / 0$ & $96.25 / 3.75 / 0$ & $96.25 / 3.75 / 0$ \\
\hline \multicolumn{4}{|l|}{ Mean B-factors $\left(\AA^{2}\right)$} \\
\hline Protein & 42.2 & 36.9 & 48.7 \\
\hline FAD/GLC/LMB & $33.4 /-/-$ & 28.8/47.7/- & $40.1 /-/ 61.7$ \\
\hline Water/Sulphate & $41.8 / 55.9$ & $42.7 / 85.9$ & $52.6 / 105.3$ \\
\hline \multicolumn{4}{|l|}{ Number of atoms } \\
\hline Protein & 5023 & 4902 & 5059 \\
\hline FAD/GLC/LMB & $53 /-/-$ & 53/108/- & $53 /-/ 115$ \\
\hline Water/Sulfate & $466 / 40$ & $363 / 20$ & $303 / 15$ \\
\hline
\end{tabular}


a. $\quad R_{\text {merge }}=\sum_{i} \sum_{j}\left|I_{i, j}-\left\langle I_{j}\right\rangle\right| / \sum_{i} \sum_{j} I_{i, j}$, where $i$ runs over multiple observations of the same intensity, and $j$ runs over all crystallographically unique intensities.

b. $\quad R_{\text {work }}=\sum|| F_{\text {obs }}|-| F_{\text {calc }}|| / \sum\left|F_{\text {obs }}\right| / \sum_{i} \sum_{j} I_{i, j}$, where $\left|\mathrm{F}_{\text {obs }}\right|>0 . \mathrm{R}_{\text {free }}$ is based on $5 \%$ of the data randomly selected and is not used in the refinement.

The FAD-binding domain is formed by the five-stranded parallel $\beta$-sheet A (B1, B2, B6, B10, B18) that is sandwiched between the three-stranded antiparallel $\beta$-sheet B (B7, B8, B9) and three $\alpha$-helices (H1, H9, H20); the $\beta \alpha \beta$-motif involved in FAD binding is formed by B1, H1 and B2.

The substrate-binding domain consists of the central six-stranded antiparallel $\beta$-sheet C (B5, B11, B12, B13, B14, B17), roofing the FAD-binding domain at the level of the FAD isoalloxazine ring and crowned by seven $\alpha$-helices (H8, H12, H13, H14, H15, H17, H18). We can depict $\beta$-Sheet C as a roof which delimits a deep pocket (ODH active site) by laying on a "floor" that is formed by the FAD-binding domain and exposes the catalytically active moiety (isoalloxazine ring) of the FAD cofactor to the cavity. The FAD-binding domain and the substrate-binding domain are linked through two types of connections: one consists of three structured extended segments connecting the two domains, the other one involves three helices that protrude from the substrate binding domain and lean on the surface of the FAD-binding one, and two extended two-stranded $\beta$-sheets $(\beta$-sheet $\mathrm{D}$, parallel, and $\beta$-sheet E, anti-parallel), located at the interface between domains and hooking them together.

The amino acid sequence of ODH contains three potential N-glycosylation sites, Asn38, Asn188 and Asn439, which could be predicted by the consensus sequence Asn-X-Ser/Thr and that are all visible in the crystallographic structure (see Additional file 1, Fig. 4B).

The FAD molecule is non-covalently bound to the protein and it occupies a narrow channel lined by ordered regions, mainly the loop connecting $\beta$-strand B1 and helix $\mathrm{H} 1$, part of a $\beta \alpha \beta$-motif. The electron density map of the FAD isoalloxazine ring clearly shows a distortion from planarity, which is expected for the oxidized FAD. To unequivocally point out the redox state of the cofactor, initial structure refinement trials were performed modelling either reduced (bent) or oxidized (planar) FAD; 
the resulting electron density maps is consistent with the presence of the cofactor in the oxidized conformation, despite the distortion from planarity. In fact, the pyrimidine moiety is $\sim 11^{\circ}$ bent toward the FAD-binding domain with respect to the pteridine plane. This bent conformation of the oxidized FAD has already been observed in other GMC oxidoreductases, where the protein backbone architecture in the proximity of FAD, and in particular a conserved asparagine, restrains the cofactor geometry, causing a distortion from planarity of the isoalloxazine ring [18-20, 26]. This interpretation is also valid for ODH, where Asn97, a conserved residue within AA3 enzymes from the GOX/GDH clade (see Additional file 1, Fig. S4), points toward the central part of the isoalloxazine ring, sticking out from the "floor" of the active site cavity (FAD-binding domain). Asn97 establishes a hydrogen bond with Ser573, conserved in some GOX/GDH subclades (see Additional file 1, Fig. S4), and hydrogen bonds with FAD and ODH backbone atoms. Preference for short side chain residues in the three positions Gly98, Ala99 and Ala100 is also observed within the GOX/GDH clade (see Additional file 1, Fig. S4), possibly allowing stabilization of the bent pyrimidine moiety through hydrogen bonding with backbone atoms (see Additional file 1, Fig. S5). A bent conformation of oxidized FAD is thought to allow switching from the oxidized to the reduced form with minor conformational rearrangements, thus resulting in a reduced energy difference between the two states and in the modulation of FAD redox potential to favor reduction.

$\mathrm{ODH}$ active site is located at the bottom of a large funnel-shaped cavity and it is directly accessible to the solvent. As in other GMC oxidoreductases, we identified the catalytic pair His528/His571, positioned in the proximity of $\mathrm{FAD}$, on the re-face of the isoalloxazine ring. The imidazole rings of His528 and His571 are oriented and stabilized by hydrogen bonds with Gln329 and Glu414 respectively, conserved within the GOX/GDH clade (see Additional file 1, Fig. S4), and with the FAD reactive N5 atom through a shared water molecule (see Additional file 1, Fig. S5). Except for these histidines and Gln331, most of the residues contributing to the active site cavity are either hydrophobic or possess aromatic side chains, such as Tyr64, Phe416 and Trp430 (see Additional file 1, Fig. S5). 


\section{Structures of ODH bound to substrates: the substrate-binding loop}

Structures of ODH-LMB (PDB entry 6XUV) and ODH-GLC (PDB entry 6XUU) were obtained by soaking and refined up to 1.75 and $1.57 \AA$ resolution respectively (Table 2). Overall, ODH-sugar complexes show very few structural differences compared to the ligand-free form: pairwise superposition onto the structure of ligand-free ODH yields average C $\alpha$ r.m.s.d. values of $0.41 \AA$ and $0.34 \AA$ respectively. The analysis of r.m.s.d. as a function of the residue number, however, shows $\mathrm{C} \alpha$ atoms displacements exceeding $10 \AA$ for residues forming the B13-B14 turn, to which we refer as the “substrate-binding loop" (residues 419 to 424, Fig. 5). While this region points toward the solvent in ligand-free ODH contributing to a wide-open active site, in sugar-bound ODH the "substrate-binding loop" restricts the active site access, bending inwards (Fig. 5). This pronounced conformational change consists of a $90^{\circ}$ bending around two hinges (Gly420 and Asp424), that allow backbone reorientation around fixed $\mathrm{C} \alpha$ atom positions. It results into a large displacement toward the interior of the active site of three amino acids (Phe421, Pro422 and Asp423), whose tight geometry is maintained in both conformations, probably relying on the cis-proline at the position 422 . Notably Phe421, exposed to the solvent in ligand-free ODH, moves $\sim 17 \AA$ towards the inside of the active site upon sugar binding, clamping either LMB non-reducing glucosyl unit or a GLC molecule against Tyr64 (Fig. 6). For more details about residues and interactions contributing to substrate-binding loop conformations see Additional File 2, Section 1. Within the GOX/GDH clade, the substrate-binding loop seems to be conserved in the ODH and ODH-like subclades from Basidiomycetes, with different loop structures in the Ascomycete subclades (see Additional file 1, Fig. S4).

\section{Structures of ODH bound to substrates: the active site}

No significant conformational changes are observed in other residues lining the active site. In the sugar-bound forms the FAD isoalloxazine ring shows a more pronounced distortion from planarity, with a bending angle of $\sim 16^{\circ}$ between the pyrimidine and the pteridine moieties (instead of $\sim 11^{\circ}$ 
observed in the ligand-free form) (Fig. 5), which may be an indication of the cofactor getting reduced upon crystal soaking with the oxidizable substrates.

A total of four LMB molecules are found in the structure of ODH-LMB: one in the active site (Fig. 6) and three on the protein external surface (Fig. 7). Similarly, the structure of ODH-GLC shows seven GLC molecules bound to the protein: four in the substrate-binding cavity (see Additional file 1, Fig. S7) and three on the protein surface (see Additional file 1, Fig. S8). In the case of ODH-GLC an additional GLC molecule (not shown) was identified at the interface between symmetry-related ODH molecules. This GLC ligand does not occupy a cavity and no LMB was found in the corresponding region of ODH-LMB, and it is likely to participate only to crystal contacts. Two GLC molecules closely mimic the binding of LMB to ODH active site: one (GLC1) is oriented as LMB reducing end, the other (GLC2) as the disaccharide non-reducing end pyranose (Fig. 6). Interestingly, in ODH-GLC two additional GLC molecules (GLC3 and 4) bind at the periphery (entrance) of the substrate-binding cavity and they could map a substrate diffusion pathway from the bulk to the FAD, suggesting that the external periphery of the substrate-binding tunnel might act as a funnel conveying substrate molecules to the reaction center (see Additional file 1, Fig. S7).

The electron density maps in the vicinity of the FAD cofactor accounting for bound LMB and GLC1 suggested that both anomeric forms of the saccharides bind to ODH active site. As such, we refined both the $\alpha$ and the $\beta$ anomers which respectively appears to display a with $35 \%$ and $65 \%$ occupancy for both LMB and GLC1. The LMB and GLC1 $\beta$ anomers are oriented in such a way that the reactive sugar $\mathrm{O} 1$ hydroxyl is hydrogen bonded to the N5 atom of the cofactor and the Nع2 atom of His528 (Fig. 6 and see Additional file 1, Fig. S6), in a position compatible for the proton abstraction hypothesized in the catalytic mechanism [18-22]. For both substrates, $\beta$ anomers are also stabilized by hydrogen bonds with Val526 backbone carbonyl (O2 hydroxyl), with Gln331 side chain (O2 and $\mathrm{O} 3$ hydroxyls) and with the O4F atom of the pyrimidine portion of FAD (O6 hydroxyl). Apart from the latter, all hydrogen bonds with ODH are lost for LMB and GLC1 $\alpha$ anomers (see Additional file 1, Fig. S6), since it is slightly shifted compared to the corresponding $\beta$ anomer (Fig. 6). Notably, $\alpha$ 
anomer $\mathrm{O} 1$ hydroxyls do not interact with the catalytic histidine and the cofactor (see Additional file 1, Fig. S6), suggesting that the presence of $\alpha$ anomers within the active site does not favor a catalytic activity from ODH. Although an additional hydrogen bond links Asp418 seems to represent key residues in $\mathrm{ODH}$ substrate binding as evidenced by sequence alignment and evolutionary conserved residues (see Additional file 2, Section 2).

Instead, both LMB and GLC $\alpha$ and the $\beta$ anomers are stabilized by $\mathrm{CH}-\pi$ interactions, established between aromatic $\pi$-systems (protein sidechains) and the sugar $\mathrm{C}-\mathrm{H}$ bond. The two side chains of Trp430 and Phe416 lie almost on the same plane, forming an aromatic platform above the $\beta$-face of LMB reducing end and GLC1 (Fig. 6, and see Additional file 1, Fig. S6). These two amino acids account for the binding of both LMB and GLC anomers, and possibly discriminate between the two anomers (for more details see Additional file 2, Section 3). This aromatic platform is extended also above the $\beta$-face of LMB non-reducing unit. The disaccharide end and GLC2 are in fact engaged in $\mathrm{CH}-\pi$ interactions with Phe421, belonging to the substrate-binding loop (see Additional file 1, Fig. S6). Phe421phenyl ring lies parallel to the non-reducing pyranose ring (Fig. 6). On the other side of ligands, the aromatic ring of Tyr64 stabilizes the $\alpha$ faces of LMB glucosyl units and GLC in both anomeric forms (see Additional file 1, Fig. S6). Despite it was proposed that Tyr64 may bind GLC in AfGDH, through canonical hydrogen bonds between substrate and tyrosine hydroxyls [20], in the light of the results reported herein and previously, the role of this tyrosine residue might be more puzzling to decipher (for more details see Additional file 2, Section 4).

To summarize, structural analysis of ODH revealed two main contributions to substrate binding in the proximity of the cofactor: i) $\mathrm{CH}-\pi$ stabilization due to electron-rich aromatic residues (Phe416, Phe421 and Trp430) and ii) the steric effects of these and other hydrophobic residues, including Tyr64, that impose a directionality to LMB and GLC molecules, binding close to FAD within a funnel-shaped tunnel (GLC1 and 2, Fig. 6 and see Additional file 1, Fig. S7), which provides the perfect environment to bind $\beta(1 \rightarrow 3)$ oligosaccharides. 


\section{Structures of ODH bound to sugars: external sugar-binding sites}

A peculiar feature of sugar-bound ODH structures is the presence of three sugar-binding sites (SBSs) on the external protein convex side, distant from the active site entrance, loceted on the concave side (Fig. 7). Their position and the details of the sugar-protein interactions are shown for ODH-LMB in Fig. 7 (see Additional file 1, Fig. S8 for ODH-GLC). LMB molecules bind to these sites through their non-reducing units, mainly contributing to SBSs interactions, while the reducing ones are exposed to the bulk and does not seem to participate to SBSs binding. GLC molecules bind in the same fashion and are superimposable to the non-reducing units of LMB. Both ligands show well-defined electron densities and B-factors comparable to those of the surrounding protein atoms, suggesting a tight binding.

SBS1 is a small pocket with residues from $\beta$-sheet $\mathrm{D}$, from helix $\mathrm{H} 7$ and from the protruding loop which connects B5 and H9. Most of the sugar-protein contacts form a dense network of hydrogen bonds involving sugar hydroxyl groups: the $\mathrm{O} 2$ atom is stabilized by Ser141 sidechain hydroxyl and by Ser141 and Ala142 backbone carbonyls; the $\mathrm{O} 3$ atom by Thr168 and Gln147 side chains, by the carbonyl group of Phe169 and by Asp219 side chain through a water molecule; finally, the O4 atom interacts with Asp219 though the same water molecule and to Asp171 sidechain. The reducing unit of LMB does not contribute significantly to binding, as it only interacts with Ala142 peptide carbonyl group through the $\mathrm{O} 2$ hydroxyl. The sugar molecules in SBS1 are further stabilized by the aromatic side chain of Trp215, engaged in $\mathrm{CH}-\pi$ interactions with the $\alpha$-face of the sugar ring.

SBS2 is located between $\mathrm{H} 1$ and $\mathrm{H} 9$, right below $\beta$-sheet D. As in SBS1, several interactions between the sugar molecules and the protein environment could be identified: GLC O2 atom is stabilized by hydrogen bonds with Glu37, Arg236 and a water molecule; the $\mathrm{O} 3$ atom also binds to Arg236 through the same water molecule, and to Tyr228 backbone carbonyl; the $\mathrm{O} 4$ hydroxyl group is involved in hydrogen bonds with the two peptide carbonyl groups of Tyr228 and Ala227. In the case of LMB, an additional hydrogen bond between the oxygen atom of the LMB $\beta(1 \rightarrow 3)$ glycosidic bond and the 
side chain of Arg34 is observed. As in SBS1, the reducing glycosyl unit of LMB provides a minor contribution to ligand binding, in this case through a hydrogen bond between the $\mathrm{O} 2$ atom and Glu37. Unlike in SBS1, no CH- $\pi$ stabilization was observed in SBS2.

SBS1 and SBS2 are close to each other, while SBS3 is further away from the active site entrance and is located between helices H8 and H12. Sugars in SBS3 show higher temperature factors (B-factors) than in SBS1 and 2 and interact with the protein through three hydrogen bonds: the $\mathrm{O} 3$ and $\mathrm{O} 4$ sugar atoms interact with the peptide carbonyl group of Thr190 and Gln359 respectively, while the O6 atom interacts with Lys476 side chain. In the case of LMB, one hydrogen bond is also found between the $\mathrm{O} 2$ atom of LMB reducing end and Ser363 side chain and backbone amide.

\section{Discussion}

\section{Role of ODH in lignocellulose breakdown}

Functional data show that the optimal substrates of ODH are sugars endowed with a next to their reducing glucosyl unit, among which LMB being the preferred one among the compounds tested. This $\beta(1 \rightarrow 3)$ glycosidic bond is found in $\beta(1 \rightarrow 3)$ glucans, a major components of the fungal cell wall [27], in bacterial curdlan [28], as well as in $\beta(1 \rightarrow 3)$ callose and in mixed $\beta(1 \rightarrow 3,1 \rightarrow 4)$ glucans typically present in the plant cell wall. Mixed glucans are part of the hemicellulose matrix and assures plant cell wall growth and integrity together with cellulose, pectin, lignin and other compounds [29, 30]. Like other plant cell wall components, hemicelluloses are fully degraded by fungi and other organisms. As for cellulose, this is achieved through the action of glycoside hydrolases (GHs), such as enzymes belonging to the GH12 and GH45 CAZy families [31], which show endo-glucanase activity toward mixed $\beta(1 \rightarrow 3,1 \rightarrow 4)$ glucans. Despite the enzymatic specificity in terms of glycosidic linkage, we observed that ODH is able to accept electrons from oligosaccharides displaying different degrees of polymerization, provided that a $\beta(1 \rightarrow 4)$ follows a $\beta(1 \rightarrow 3)$ glycosidic bond, counting from the reducing-end of the oxidizable saccharide. In the light of these observations, we can speculate on 
the physiological role of ODH. Among all possible oligosaccharides released by the cleavage of mixed $\beta(1 \rightarrow 3,1 \rightarrow 4)$ glucans, ODH is able to oxidize GLC, LMB and GTB (Fig. 8). CLB also produced in large amount by fungal cellulolysis, cannot be oxidized by ODH but by the flavodomain (CAZy subfamily AA3_1) of CLB dehydrogenase (CDH). Electron deriving from CLB oxidation are used to fuel the lytic polysaccharide monooxygenase (LPMO) activity [32]. Completion of the LPMO catalytic cycle requires in fact a $\mathrm{Cu}$ (II) reduction step, that can also be sustained in vitro by diffusible redox mediators which are reduced by AA3_2 dehydrogenases, such as ODH [33, 34]. AA3_2 dehydrogenases might also reduce phenoxy radicals and assist laccases (CAZy family AA1) in lignin degradation $[13,14]$, whereas AA3_2 oxidases would produce hydrogen peroxide, a co-substrate for LPMOs and fungal peroxidases (CAZy family AA2) [35, 36]. Many other enzymes play similar roles in fungal lignocellulose breakdown. A notable example are AA7 flavoenzymes from Ascomycetes, which have been shown to oxidase cellulose-derived oligosaccharides[37], hemicellulose-derived xylo-oligosaccharides[38] and even polysaccharides, including $\beta$-glucans[39]. Within this scenario, the identification of $\mathrm{ODH}$ activity points towards another source of oligosaccharides, containing $\beta(1 \rightarrow 3)$ glycosidic bonds and derived from hemicellulose ( $\beta$-glucans), within the palette of compounds that fungi can oxidize to maintain redox homeostasis of lignocellulose-degrading enzymes. These observations can be interpreted as an enzymatic adaptation to substrate availability and they expand the range of known sugars derived from hemicelluloses that can be oxidized by Basidiomycetes. Activity on oligosaccharides is not unique to ODH, as it has also been reported for $\mathrm{CDH}$ and AA7 enzymes, although regarding different substrates, which, in either cases, allows the oxidation of only partially deconstructed polysaccharide. Finally, the GOX/GDH clade of AA3_2 enzymes seems to include enzymes with different physiological functions: monosaccharide oxidoreductases, such as GOX and GDH from Ascomycetes, and oligosaccharide oxidoreductases, such as ODH, that seem more efficient in transforming plant cell wall components, as expected for saprophytic and pathogenic Basidiomycetes. 


\section{Structure-function analysis of $\mathrm{ODH}$}

The three crystallographic structures herein reported, provide a molecular explanation for ODH substrate specificity. Preferred activity towards a disaccharide is structurally justified by the recruitment of Phe421 to stabilize the non-reducing glucosyl unit, owing to the conformational change of the substrate-binding loop. Interestingly, this rearrangement occurs also when GLC binds to $\mathrm{ODH}$ active site, where two molecules of GLC mimic the disaccharide (LMB) binding geometry. As such, we can not exclude that the substrate-binding loop might also play a role in escorting monosaccharides toward the active site and, indeed, the presence of four GLC molecules to ODH active site and funnel-shaped tunnel in ODH-GLC suggests the possible existence of a well-defined substrate diffusion pathway.

The main forces responsible LMB and GLC orientation in ODH active site are $\mathrm{CH}-\pi$ interactions with aromatic residues, rather conserved in ODH-related enzymes. On the contrary, in GDH class-I and GOX enzymes, such as AfGDH (PDB: 4YNT [20]) and AnGOX (1CF3 [19]), the molecular determinants for the formation of the sugar-enzyme complex rely on specific hydrogen bonds between sugar hydroxyl groups and conserved polar residues from the active site (see Additional file 1, Fig. S4 and S6).

To our knowledge, the presence of secondary SBSs on the enzyme surface has not yet been reported for other GMC oxidoreductases. However, they seem to be conserved to a certain extent within the GOX/GDH clade, particularly SBS1 and 2 (see Additional file 1, Fig. S4). The molecular bases of sugar recognition in these sites restrict the hypothetical natural binders to a limited group of carbohydrates. Only monosaccharides and oligosaccharide non-reducing ends bound to the SBSs in ODH crystallographic structures. Potential, naturally occurring ligands may be found among nonreducing ends of polysaccharides, like cellulose or hemicellulose, and of their cleavage products. Interestingly, branched polysaccharides in hemicellulose, such as xyloglucans, glucuronoxylans, galactomannan and galactoglucomannans, are plausible binders too. The xylose, glucuronic acid and galactose units, forming the branches of these polysaccharides, possess in fact free $\mathrm{O} 2, \mathrm{O} 3$ and $\mathrm{O} 4$ 
hydroxyl groups [29] with identical stereochemistry identical to GLC, except for the galactose O4 hydroxyl group. If the external SBSs identified in ODH are able to recognize branched polysaccharides, they might have a physiological role, as they may anchor ODH and possibly other AA3 enzymes to specific polysaccharide domains of lignocellulose where to drive their catalytic activity.

\section{Conclusions}

We identified oligosaccharides that contain $\beta(1 \rightarrow 3)$ glycosidic bonds, such as LMB, as preferred enzyme substrates, and we provided an explanation for enzyme promiscuity towards GLC. Indeed, $\mathrm{ODH}$ activity on monosaccharides previously led to the definition of GDH class-III enzymes. However, in the light of the results described herein we redefined the investigated enzyme as an oligosaccharide dehydrogenase (ODH). The ability to accept electrons from mono- and oligosaccharides deriving from hemicellulose breakdown is consistent with ODH being an efficient enzymatic tool for saprophytic and pathogenic Basidiomycetes, which need to master lignocellulose deconstruction for their survival.

The structure of ODH, the first characterized enzyme within the ODH subclade of AA3_2 enzymes, represents a major step toward understanding the enzymatic diversity within the GOX/GDH clade as it represents the first structure of such an enzyme derived from a Basidiomycete and the second structure of a dehydrogenase from the GOX/GDH clade. Structure-function analysis points out some novel, unexpected features of ODH substrate recognition, especially: i) the lack of specific glucosebinding residues (as found instead in GDH class-I and GOX) and the presence of three key aromatic residues (Phe416, Phe421 and Trp430 in ODH); ii) the involvement of a highly flexible substratebinding loop in the process of substrate recognition; iii) the presence of external SBSs, which might play a role in sensing substrate availability and directing the enzymatic activity toward the polysaccharide matrix, thus contributing to the overall enzymatic efficiency. ODH characterization is a good example of extended versatility, even within a small group of phylogenetically related 
enzymes such as the GOX/GDH clade of AA3_2 enzymes. As seen from previous phylogenetic analysis, increased biodiversity supported by novel, uncharacterized and unexpected enzymatic functions is yet to be expected within the AA3_2 subfamily of GMC oxidoreductases, including about 10 phylogenetic (sub)clades of enzymes of unknown function.

Auxiliary enzymes involved in lignocellulose degradation, like ODH, represent promising candidates for the development of novel biotechnologies in the context of the plant biomass biorefinery. Indeed, ODH could fuel LPMO enzymatic activity and inactivate laccase-generated phenoxy radicals, improving as such the breakdown of recalcitrant cellulose and inhibiting lignin repolymerization during biomass enzymatic saccharification. The preference of ODH for oligosaccharides containing $\beta(1 \rightarrow 3)$ glycosidic bonds implies that $\mathrm{ODH}$ and related enzymes might be particularly useful and effective in treating specific types of biomass. This is the case for plants that are naturally rich in mixed $\beta(1 \rightarrow 3,1 \rightarrow 4)$ glucans, such as those belonging to the Poales order, which includes extensively cultivated plant species wheat, rice and corn, amongst others and accounts for a large fraction of poorly valorized agro-industrial residues.

\section{Methods}

\section{Chemicals}

2,6-dichlorophenolindophenol (DCIP or DCPIP), GLC, GAL, XLS, LAC, MAN, MAL and CLB were purchased from from MilliporeSigma (Burlington, MA, USA). LMB, LMT, LMTT, GTA, GTB, CLT, CLTT were purchased from Megazyme (Bray, Ireland).

\section{Crystallization and crystal handling}

ODH was expressed and purified as described in Piumi et al. [14]. Purified ODH was exchanged, after IMAC Ni-NTA affinity chromatography, in buffer with no imidazole and concentrated to 17.4 $\mathrm{mg} \mathrm{mL}^{-1}$ for crystallization trials. ODH concentration was measured using a NanoDrop ${ }^{\mathrm{TM}} 2000$ (Thermo Fisher Scientific, Waltham, MA, USA), assuming an extinction coefficient $\varepsilon_{280}=89840 \mathrm{M}^{-1}$ $\mathrm{cm}^{-1}\left(\mathrm{Abs}_{280} 0.1 \%=1.510\right)$ estimated from ODH amino acid sequence using ExPASy Protparam 
[40]. Crystallization plates were initially set up automatically using a Crystal Phenix robot (Art Robbins Instruments, Sunnyvale, CA, USA) following the in sitting-drop vapor-diffusion method at 294 K using Intelli-plate 96 well (Art Robbins Instruments, Sunnyvale, CA, USA). Single crystals were obtained using the AmSO4 suite (Qiagen, Hilden, Germany) crystallization screen. The reproducibility of crystallization conditions was tested setting up hand-made plates using the hangingdrop vapor diffusion method at $294 \mathrm{~K}$, which led to the formation of larger crystals. Well-diffracting single crystals grew by mixing $1 \mu \mathrm{L}$ of protein solution and $1 \mu \mathrm{L}$ of reservoir solution containing 2 $\mathrm{M}\left(\mathrm{NH}_{4}\right)_{2} \mathrm{SO}_{4}$ and equilibrating the droplet against $0.5 \mathrm{~mL}$ of reservoir solution. Cryoprotection was achieved by transferring ligand-free ODH crystals in a solution containing $2 \mathrm{M}\left(\mathrm{NH}_{4}\right)_{2} \mathrm{SO}_{4}$ and $2 \mathrm{M}$ $\mathrm{LiSO}_{4}$, which were then flash-frozen in liquid nitrogen. ODH-GLC crystals were prepared by soaking ligand-free $\mathrm{ODH}$ crystals in a solution containing $2 \mathrm{M}\left(\mathrm{NH}_{4}\right)_{2} \mathrm{SO}_{4}$ and $80 \%$ GLC which also acted as cryoprotectant; after about 12 min incubation, crystals were flash-frozen in liquid nitrogen. Analogously, ODH-LMB crystals were obtained through 20 min soaking for in a solution containing $2 \mathrm{M}\left(\mathrm{NH}_{4}\right)_{2} \mathrm{SO}_{4}$ and $8 \% \mathrm{LMB}$. Crystals were cryoprotected in $2 \mathrm{M}\left(\mathrm{NH}_{4}\right)_{2} \mathrm{SO}_{4}$ and $2 \mathrm{M} \mathrm{LiSO}_{4}$, and flash-frozen in liquid nitrogen prior to data collection at synchrotron radiation sources.

\section{Structure determination and refinement}

X-ray diffraction data of ligand-free ODH and ODH-LMB were collected at the Diamond synchrotron (Harwell, UK), beamline I24; data for ODH-GLC were collected at ELETTRA (Trieste, Italy), beamline XRD2. All datasets were collected at $100 \mathrm{~K}$ using a PILATUS detector. Data were indexed, scaled and integrated using the XDS package [41]. Molecular replacement was carried out using MOLREP [42] from the CCP4 suite [43]. The structure of $A f G D H$ at $1.78 \AA$ resolution (PDB entry 4YNT [20], 36.4\% sequence identity to ODH) was used as search model to calculate the initial crystallographic phases of ligand-free ODH, whose structure was then employed to obtain the initial phases for both ODH-GLC and ODH-LMB. Iterative structure refinement and model building were carried out using REFMAC5 [44] and COOT [45] respectively, both implemented in the CCP4 suite. The Translation-Libration-Screw-rotation (TLS) model of rigid-body harmonic displacements was 
included during the last cycles of automated refinement [46]. 5\% of the reflections were excluded from refinement for cross validation by means of the free R-factor [47]. Manual model building was performed based on the $F_{0}-F_{c}$ map contoured at $3 \sigma$ and the $2 F_{0}-F_{c}$ map at $1 \sigma$. Validation of the models, including Ramachandran statistics and B-factor analysis, was carried out using the Multimetric model geometry validation tool implemented in the CCP4 suite. Protein sequence alignment was performed using Clustal Omega [48], and structural superposition using Superpose [49], implemented in the CCP4 suite. Molecular graphics figures were produced using Chimera [50].

\section{Spectrophotometric assays for enzymatic activity}

Functional assays have been carried out using DCIP as the secondary electron acceptor in the half reaction responsible for FAD oxidation [14]. Enzymatic activity was evaluated spectrophotometrically monitoring the loss in DCIP absorbance upon reduction at $520 \mathrm{~nm}(\Delta \mathrm{Abs} 520$, $\varepsilon_{520}=6800 \mathrm{M}^{-1} \mathrm{~cm}^{-1}$ ), which represents a $\mathrm{pH}$-independent isosbestic point [51].

All spectrophotometric assays were performed using a sample final volume of $0.1 \mathrm{~mL}$ in a $0.2 \mathrm{~mL}$ 96-well plate (Corning Costar, Corning, NY, USA) using a Multiskan GO microplate spectrophotomer (Thermo Scientific, Waltham, MA, USA) in static (substrate screening) and in kinetic (enzyme kinetics) modes. Plates and solutions without the enzyme were first equilibrated at 303.15 K $\left(30{ }^{\circ} \mathrm{C}\right)$; upon addition of $\mathrm{ODH}$, solutions were automatically mixed and the signal at 520 nm was recorded. Each measurement was taken at least in triplicate independent experiments. The absorbance signal of DCIP was converted into concentration units by comparison to a calibration curve (Abs520 vs [DCIP]) measured concomitantly to each experiment. The slope of a linear fit of Abs520 vs [DCIP] was used to calculate $\Delta[\mathrm{DCIP}]$ in all experiments.

The pH-dependence of ODH enzymatic activity was assessed using $720 \mathrm{mM}$ GLC and $0.4 \mathrm{mM}$ DCIP either in $50 \mathrm{mM}$ citrate-phosphate buffer $\mathrm{pH} 3.5,4.5,5.5,6.5$ and 7.5, or in $50 \mathrm{mM}$ Tris $\cdot \mathrm{HCl} \mathrm{pH}$ 8.5. To start the reaction ODH was added at a final concentration of $39.7 \mathrm{nM}$. Initial velocities were measured spectroscopically by monitoring the rate of the absorbance decay at $520 \mathrm{~nm}$ over time for a total of 20 minutes. Initial rates were estimated by applying a linear fit to the linear region of the 
time trace. Optimal $\mathrm{pH}$ fell in the range 5.5-6, as previously reported [12]. All remaining spectrophotometric assays were carried out at in $0.4 \mathrm{mM}$ DCIP and $50 \mathrm{mM}$ citrate-phosphate buffer $\mathrm{pH} 5.5$.

ODH substrate screening was performed using fourteen different sugar compounds: GLC, XLS, GAL, MAN, LAC, MAL, CLB, LMB, CLT, LMT, GTA, GTB, CLTT and LMTT (see Additional file 1, Fig. S1). Because of different solubility, all sugar substrates were employed at a fixed concentration of $2.5 \mathrm{mM}$, with the exception of GLC that was also used at $250 \mathrm{mM}$ as a positive control of $\mathrm{ODH}$ reaction. The enzyme was added at a final concentration of $39.7 \mathrm{nM}$ to start the reaction ( $\mathrm{t}=0$ ). DCIP reduction was followed over the time by acquiring triplicates of the Abs520 after an incubation of 2 and 5 minutes, as well as 3 and 19 hours. Each data point was collected on three identical, independently prepared reaction mixtures.

The same experimental set-up was used to evaluate the kinetic parameters of ODH using GLC and LMB as substrates. GLC and LMB were tested at different concentrations ranging from 0 to 3000 $\mathrm{mM}$ and from 0 to $115.5 \mathrm{mM}$, respectively. For each substrate concentration initial velocities were measured spectroscopically by monitoring the rate of absorbance decay at $520 \mathrm{~nm}$ over time for a total of 20 minutes in triplicate experiments. Initial rates were estimated by fitting the linear region of the time trace. Initial velocities measured at various substrate concentrations were fitted, using the Kaleidagraph software package, to the hyperbolic equation of Michaelis-Menten:

$$
V=\frac{V_{\max }[S]}{K_{M}+[S]}
$$

where $\mathrm{V}$ is the initial velocity of the reaction, $\mathrm{V}_{\max }$ is the maximum velocity of the reaction corresponding to the substrate saturation of the enzyme, $\mathrm{K}_{\mathrm{M}}$ is the Michaelis-Menten constant and [S] is the substrate analytical concentration. The Michaelis-Menten equation was applied to either all concentration points (LMB) or to points that looked unaffected by inhibition at extremely high substrate concentrations (GLC), as better detailed in the Results section.

\section{Analysis of ODH reaction products by LC-MS}


Liquid Chromatography was performed on a UHPLC Ultimate 3000RS (Thermo Scientific) coupled to a Charged Aerosol Detector (CAD Corona, Thermo Scientific) and an ISQ-EM mass spectrometer with heated ESI-interface (Thermo Scientific). The eluent was split 1:1 and the resulting flow from the LC to the MS was in all cases $0.125 \mathrm{~mL} / \mathrm{min}$. The heated ESI was operated at $348 \mathrm{~K}$ in negative mode at $-2 \mathrm{kV}$ spray current, with a sheath gas flow of 23.5 and an auxiliary gas flow of 2.6 (arbitrary units). The capillary temperature was $523 \mathrm{~K}$. UHPLC-ESI-MS data were acquired and analyzed with the Chromeleon software v7.2.10 (Thermo Scientific). An Acquity UHPLC BEH Amide column (2.1 mm x $150 \mathrm{~mm}, 1.7 \mathrm{~mm}$, Waters, Milford, USA) was used for chromatographic separation of analytes. $\mathrm{ODH}$ enzymatic assays were performed on GLC and LMB in order to detect and identify the enzymatic products. Reaction mixtures were prepared in unbuffered water and contained $37.5 \mathrm{mM}$ substrate, $45 \mathrm{mM}$ DCIP and $90 \mathrm{nM}$ ODH. Reactions were run for 21 hours at $348 \mathrm{~K}$. Enzymatic assay aliquots were diluted 5 times in acetonitrile $(20 \mu \mathrm{L}$ enzymatic assay $+80 \mu \mathrm{L}$ acetonitrile $)$ and $2 \mu \mathrm{L}$ of the diluted samples were injected. The column temperature was maintained at $30^{\circ} \mathrm{C}$. The isocratic elution method used ammonium formate $12 \mathrm{mM}$-acetonitrile $(35 \% / 65 \% \mathrm{v} / \mathrm{v})$ at a flow rate of $0.25 \mathrm{~mL}$ $\mathrm{min}^{-1}$. The mass range from 50 to $1500 \mathrm{~m} / \mathrm{z}$ was monitored.

\section{Abbreviations}

AfGDH: glucose dehydrogenase from A. flavus; AnGOX: glucose oxidase from A. niger; CAZy: carbohydrate-active enzymes; CLB: cellobiose; CLT: cellotriose; CLTT: cellotetraose; DCIP: 2,6dichlorophenolindophenol; FAD: flavin adenine dinucleotide; GAL: D-galactose; GDH: glucose dehydrogenase; GLC: D-glucose; GMC: glucose-methanol-choline; GOX: glucose oxidase; GTA: 1,3;1,4 $\beta$-glucotriose A; GTB: 1,3;1,4 $\beta$-glucotriose B; LAC: D-lactose; LGC: D-glucono- $\delta$-lactone; LMB: laminaribiose; LMT: laminaritriose; LMTT: laminaritetraose; MAL: D-maltose; MAN: Dmannose; ODH: oligosaccharide dehydrogenase; ODH-GLC: structure of ODH in complex with glucose (PDB: 6XUU); ODH-LMB: structure of ODH in complex with laminaribiose (PDB: 6XUV); 
PDB: protein data bank; r.m.s.d.: root-mean-square deviation; SBS: sugar-binding site; XLS: Dxylose.

\section{Declarations}

Ethics approval and consent to participate

Not applicable

\section{Consent for publication}

Not applicable

\section{Availability of data and materials}

The datasets used and/or analyzed during the current study are available from the corresponding author on reasonable request. Coordinates and mtz files for the crystallographic structures have been deposited in the Protein Data Bank (PDB) with accession codes 6XUT (ligand-free ODH), 6XUU (ODH-GLC) and 6XUV (ODH-LMB).

\section{Competing interests}

The authors declare no competing interests.

\section{Funding}

This study was supported by an ANS grant from the "Transform" INRAe Department to G. Sciara (2018-19), by the French National Research Agency (ANR) grants FUNCLIPRO (ANR-19-CE430007-01) and FUNLOCK (ANR-13-BIME-0002-01) and by the Regional grant CIRM-CF 2.0 (APR Platforms PACA Region 2017). This work was supported by grants from Sapienza: Grant RM118164227A5FD6, Grant RM11916B838A3C38, 2018 and 2019 grants Sapienza Avvio alla Ricerca to GC and to IF respectively.

\section{Authors' contributions}

GS, BV, CS and ER conceived the study; GC, EG, LCM, CS, BV, AL and GS planned the experiments, interpreted the data, and wrote the manuscript; GC, LCM, CS, BV, DC and GS crystallized $\mathrm{ODH}$ and determined the crystallographic structures. GC, LCM, DC, CE and IF 
performed soaking experiments for ODH bound to sugars; EG, ATD, AL and GS carried out the substrate specificity and kinetic analysis of ODH. DN performed LC-MS analysis.

\section{Acknowledgements}

We acknowledge Elettra Sincrotrone Trieste for providing access to its synchrotron radiation facilities and we thank Nicola Demitri and Annie Heroux for assistance in using beamline XRD2. The authors would like to thank Diamond Light Source for beamtime and the staff of beamlines I24 for assistance with crystal testing and data collection, and synchrotrons Soleil (FR) and ESRF (FR) for the beamtime granted for crystal diffraction screening. Support from the H2CU Center to BV is acknowledged.

\section{References}

1. Janusz G, Pawlik A, Sulej J, Swiderska-Burek U, Jarosz-Wilkołazka A, Paszczynski A. Lignin degradation: microorganisms, enzymes involved, genomes analysis and evolution. FEMS Microbiol Rev. 2017;41:941-62.

2. Kirk TK, Farrell RL. Enzymatic "combustion": the microbial degradation of lignin. Annu Rev Microbiol. 1987;41:465-505.

3. Levasseur A, Drula E, Lombard V, Coutinho PM, Henrissat B. Expansion of the enzymatic repertoire of the CAZy database to integrate auxiliary redox enzymes. Biotechnol Biofuels. 2013;6:41.

4. Cantarel BL, Coutinho PM, Rancurel C, Bernard T, Lombard V, Henrissat B. The Carbohydrate-Active EnZymes database (CAZy): an expert resource for Glycogenomics. Nucleic Acids Res. 2009;37:D233-8.

5. Levasseur A, et al. The genome of the white-rot fungus Pycnoporus cinnabarinus: a basidiomycete model with a versatile arsenal for lignocellulosic biomass breakdown. BMC Genomics. 2014; 15:486.

6. Lomascolo A, Uzan-Boukhris E, Herpoël-Gimbert I, Sigoillot J, Lesage-Meessen L. Peculiarities of Pycnoporus species for applications in biotechnology. Appl Microbiol and Biotechnol. 2011;92:1129-49.

7. Estrada Alvarado I, Navarro D, Record E, Asther M, Asther M, Lesage-Meessen L. Fungal biotransformation of p-coumaric acid into caffeic acid by Pycnoporus cinnabarinus: an alternative for producing a strong natural antioxidant. World J Microbiol Biotechnol. 2003; 19:157-60.

8. Tilay A, Bule M, Annapure U. Production of Biovanillin by One-Step Biotransformation Using Fungus Pycnoporous cinnabarinus. J Agric Food Chem. 2010;58:4401-5.

9. Gross B, Yonnet G, Picque D, Brunerie P, Corrieu G, Asther M. Production of methylanthranilate by the basidiomycete Pycnoporus cinnabarinus. Appl Microbiol and Biotechnol. 1990;34:387-91.

10. Cao Y, Chen SS, Zhang S, Ok YS, Matsagar BM, Wu KC, et al. Advances in lignin valorization towards bio-based chemicals and fuels: Lignin biorefinery. Bioresour Technol. 2019;291:121878. 
11. Shraddha, Shekher R, Sehgal S, Kamthania M, Kumar A. Laccase: microbial sources, production, purification, and potential biotechnological applications. Enzyme Res. 2011;2011:217861.

12. Sygmund C, Klausberger M, Felice AK, Ludwig R. Reduction of quinones and phenoxy radicals by extracellular glucose dehydrogenase from Glomerella cingulata suggests a role in plant pathogenicity. Microbiology. 2011;157:3203-12.

13. Mathieu Y, Piumi F, Valli R, Aramburu JC, Ferreira P, Faulds CB, et al. Activities of Secreted Aryl Alcohol Quinone Oxidoreductases from Pycnoporus cinnabarinus Provide Insights into Fungal Degradation of Plant Biomass. Appl Environ Microbiol. 2016;82(8):2411-23.

14. Piumi F, Levasseur A, Navarro D, Zhou S, Mathieu Y, Ropartz D, et al. A novel glucose dehydrogenase from the white-rot fungus Pycnoporus cinnabarinus: production in Aspergillus niger and physicochemical characterization of the recombinant enzyme. Appl Microbiol Biotechnol. 2014;98:10105-18.

15. Cavener DR. GMC Oxidoreductases. A Newly Defined Family of Homologous Proteins with Diverse Catalytic Activities. J Mol Biol. 1992;223:811-4.

16. Sützl L, Foley G, Gillam EMJ, Bodén M, Haltrich D. The GMC superfamily of oxidoreductases revisited: analysis and evolution of fungal GMC oxidoreductases. Biotechnol Biofuels. 2019;12:118.

17. Sützl L, Laurent CVFP, Abrera AT, Schütz G, Ludwig R, Haltrich D. Multiplicity of enzymatic functions in the CAZy AA3 family. Applied Microbiology and Biotechnology. 2018;102:2477-92.

18. Hecht HJ, Kalisz HM, Hendle J, Schmid RD, Schomburg D. Crystal Structure of Glucose Oxidase from Aspergillus niger Refined at 2.3 A Resolution. J Mol Biol. 1993;229:153-72.

19. Wohlfahrt G, Witt S, Hendle J, Schomburg D, Kalisz HM, Hecht HJ. 1.8 and 1.9 A resolution structures of the Penicillium amagasakiense and Aspergillus niger glucose oxidases as a basis for modelling substrate complexes. Acta Crystallogr D Biol Crystallogr. 1999;55:969-77.

20. Yoshida H, Sakai G, Mori K, Kojima K, Kamitori S, Sode K. Structural analysis of fungusderived FAD glucose dehydrogenase. Sci Rep. 2015;5:13498.

21. Leskovac V, Trivić S, Wohlfahrt G, Kandrac J, Pericin D. Glucose oxidase from Aspergillus niger: the mechanism of action with molecular oxygen, quinones, and one-electron acceptors. Int $\mathrm{J}$ Biochem Cell Biol. 2005;37(4):731-50.

22. Wohlfahrt G, Trivić S, Zeremski J, Pericin D, Leskovac V. The chemical mechanism of action of glucose oxidase from Aspergillus niger. Mol Cell Biochem. 2004;260:69-83.

23. Ferri S, Kojima K, Sode K. Review of Glucose Oxidases and Glucose Dehydrogenases: A Bird's View of Glucose Sensing Enzymes. J Diabetes Sci Technol. 2011;5:1068-76.

24. Garcia-Rubio LH. Refractive index effects on the absorption spectra of macromolecules. Macromolecules. 1992;25(10):2608-13.

25. Sampedro JG, Muñoz-Clares RA, Uribe S. Trehalose-Mediated Inhibition of the Plasma Membrane H+-ATPase from Kluyveromyces lactis: Dependence on Viscosity and Temperature. J Bacteriol. 2002;184(16):4384-91.

26. Lyubimov AY, Heard K, Tang H, Sampson NS, Vrielink A. Distortion of flavin geometry is linked to ligand binding in cholesterol oxidase. Protein Sci. 2007;16:2647-56.

27. Gow NAR, Latge JP, Munro CA. The Fungal Cell Wall: Structure, Biosynthesis, and Function. Microbiol Spectr. 2017;5:3.

28. McIntosh M, Stone BS, Stanisich VA. Curdlan and other bacterial (1-->3)-beta-D-glucans. Appl Microbiol Biotechnol. 2005;68(2):163-73.

29. Scheller HV, Ulvskov P. Hemicelluloses. Annu Rev Plant Biol. 2010;61:263-89.

30. Buckeridge MS, Rayon C, Urbanowicz B, Tiné MAS, Carpita NC. Mixed Linkage (1>3),(1->4)- $\beta$-D-Glucans of Grasses. Cereal Chem. 2004;81:115-27.

31. Payne CP, Knott BC, Mayes HB, Hansson H, Himmel ME, Sandgren M, et al. Fungal Cellulases. Chem Rev. 2015;115:1308-448. 
32. Tan TC, Kracher D, Gandini R, Sygmund C, Kittl R, Haltrich D, et al. Structural basis for cellobiose dehydrogenase action during oxidative cellulose degradation. Nature Communications. 2015;6(7542):1-11.

33. Kracher D, Scheiblbrandner S, Felice AKG, Breslmayr E, Preims M, Ludwicka K, et al. Extracellular electron transfer systems fuel cellulose oxidative degradation. Science. 2016;352(6289):1098-101.

34. Garajova S, Mathieu Y, Beccia MR, Bennati-Granier C, Biaso F, Fanuel M, et al. Singledomain flavoenzymes trigger lytic polysaccharide monooxygenases for oxidative degradation of cellulose. Sci Rep. 2016;6(28276).

35. Hangasky JA, Iavarone AT, Marletta MA. Reactivity of $\mathrm{O} 2$ versus $\mathrm{H} 2 \mathrm{O} 2$ with polysaccharide monooxygenases. PNAS. 2018;115(19):4915-20.

36. Bissaro B, Várnai A, Røhr ̊̊K, Eijsink VGH. Oxidoreductases and Reactive Oxygen Species in Conversion of Lignocellulosic Biomass. Microbiol Mol Biol Rev. 2018;82(4):e00029-18.

37. Lee M-H, Lai W-L, Lin S-F, Hsu C-S, Liaw S-H, Tsai H-C. Structural characterization of glucooligosaccharide oxidase from Acremonium strictum. Appl Environ Microbiol. 2005;71(12):8881-7.

38. Vuong TV, Vesterinen A-H, Foumani M, Juvonen M, Seppälä J, Tenkanen M, et al. Xyloand cello-oligosaccharide oxidation by gluco-oligosaccharide oxidase from Sarocladium strictum and variants with reduced substrate inhibition. Biotechnol Biofuels. 2013;6(1):148.

39. Foumani M, Vuong TV, MacCormick B, Master ER. Enhanced Polysaccharide Binding and Activity on Linear $\beta$-Glucans through Addition of Carbohydrate-Binding Modules to Either Terminus of a Glucooligosaccharide Oxidase. PLoS One. 2015;10(5):e0125398.

40. Wilkins MR, Gasteiger E, Bairoch A, Sanchez JC, Williams KL, Appel RD, et al. Protein identification and analysis tools in the ExPASy server. Methods Mol Biol. 1999;112:531-52.

41. Kabsch W. XDS. Acta Crystallogr D Biol Crystallogr. 2010;66:125-32.

42. Vagin A, Teplyakov A. MOLREP: an automated program for molecular replacement. J Appl Crystallogr. 1997;30:1022-5.

43. Collaborative Computational Project 4. The CCP4 suite: programs for protein crystallography. Acta Crystallogr D Biol Crystallogr. 1994;50:7690-763.

44. Pannu N, Murshudov G, Dodson E, Read RJ. Incorporation of prior phase information strengthens maximum-likelihood structure refinement. Acta Crystallogr D Biol Crystallogr. 1998;54:1285-94.

45. Emsley P, Lohkamp B, Scott WG, Cowtan K. Features and development of Coot. Acta Crystallogr D Biol Crystallogr. 2010;66:486-501.

46. Winn M, Isupov M, Murshudov GN. Use of TLS parameters to model anisotropic displacements in macromolecular refinement. Acta Crystallogr D Biol Crystallogr. 2000;57:122-33.

47. Brunger AT. Free R value: a novel statistical quantity for assessing the accuracy of crystal structures. Nature. 1992;355:472-5.

48. Madeira F, Park YM, Lee J, Buso N, Gur T, Madhusoodanan N, et al. The EMBL-EBI search and sequence analysis tools APIs in 2019. Nucleic Acids Res. 2019;47(W1):W636-W41.

49. Krissinel E, Henrick K. Secondary-structure matching (SSM), a new tool for fast protein structure alignment in three dimensions. Acta Crystallogr D Biol Crystallogr. 2004;60:2256-68.

50. Pettersen EF, Goddard TD, Huang CC, Couch GS, Greenblatt DM, Meng EC, et al. UCSF Chimera - A visualization system for exploratory research and analysis. J Comput Chem. 2004;25:1605-12.

51. Armstrong JM. The Molar Extinction Coefficient of 2,6-Dichlorophenol Indophenol. Biochim Biophys Acta. 1964;86:194-7. 


\section{Figure titles ( $\max 15$ words) and legends}

Fig. 1 Catalytic cycle of GDHs. The enzyme in the oxidized resting state accepts electrons from a reducing sugar, like D-glucose, which is oxidized at the $\mathrm{C} 1$ position and converted into D-glucono$\delta$-lactone; this causes the protein solution to turn from yellow to colorless, due to the reduction of the FAD cofactor. In the second half-reaction electrons are transferred from reduced $\mathrm{FADH}_{2}$ to aromatic electron acceptors, such as quinones.

Fig. 2 ODH substrate screening. Histograms correspond to the amount of DCIP which is reduced over time using a set of 14 sugars as electron donors for ODH reaction. Columns are colored using a grey scale code from black to light grey ( $\mathrm{t}=2 \mathrm{~min}$ to $\mathrm{t}=19 \mathrm{~h}$, respectively). Sugar substrates were all tested at the same concentration $(2.5 \mathrm{mM})$. Negative (CTRL -) and positive (GLCx100) control reactions were conducted with no substrate and with $250 \mathrm{mM}$ GLC respectively and are reported on the left of the dashed line. Absorbance decrease at $520 \mathrm{~nm}$ was converted into percentage of reduced DCIP, using as $100 \%$ the absorbance of a solution containing no DCIP. Error bars represent the standard deviation of three independent experiments.

Fig. 3 Initial rates of ODH reaction as a function of substrate concentration. ODH enzymatic kinetic assays were performed in the presence of GLC (left panel) and LMB (right panel) at different concentrations. Data were fitted to the standard Michaelis-Menten hyperbolic equation. Insets represent a linear plot of initial velocities of $\mathrm{ODH}$ at low substrate concentrations in the linear region of the Michaelis-Menten hyperbola. Error bars represent the standard deviation of three independent experiments. 
Fig. 4 Overall structure of ODH and sequence alignment with homologous enzymes of known structure. a Sequence alignment of ODH, $A f G D H$ and $A n G O X$ based on structural superposition. Numbers refer to ODH sequence; conserved residues are colored in red. Secondary structure elements are assigned for ODH: $\beta$-strands are in orange and $\alpha$-helices in light blue. $\mathbf{b}$ Ribbon representation of the whole three-dimensional structure of ligand-free $\mathrm{ODH}$, with secondary structures assignment for $\beta$-sheets and $\alpha$-helices $(\mathrm{H})$. The FAD-binding domain is shown in yellow, the substrate-binding domain in light blue, the FAD cofactor in dark red sticks. Glycosylations are represented in sticks, with $\mathrm{C}$ atoms in yellow or light blue, $\mathrm{O}$ in red and $\mathrm{N}$ in blue.

Fig. 5 Conformational rearrangement of the substrate-binding loop upon sugar binding. Superposition of ligand-free ODH (gray) and ODH-LMB (green): a side view (perpendicular to the loop hinge axis), b top view (along the loop hinge axis). Superposition of ligand-free ODH (gray) and ODH-GLC (light blue) structures: c side view, d top view. LMB and GLC C atoms are in pink and orange respectively, $\mathrm{O}$ atoms in red and $\mathrm{N}$ atoms in blue. For clarity sake, only $\beta$ anomers are represented. Phe421 (also shown in sticks) is exposed to the bulk in the ligand free structure and shifts $\sim 17 \AA$ toward the active site upon binding of sugars, establishing $\mathrm{CH}-\pi$ interactions with the non-reducing glucosyl unit of LMB, or with GLC2 in ODH-GLC. This movement causes the substrate-binding loop to rotate by $\sim 90^{\circ}$ and wrap toward the active site.

Fig. 6 Sugar substrates bound to ODH active site. a Top view (perpendicular to the isoalloxazine plane) and b side view of ODH-LMB: the protein is shown in green, the FAD cofactor in yellow, LMB in pink ( $\beta$ anomer) or magenta ( $\alpha$ anomer). $\mathbf{c}$ Top view and $\mathbf{d}$ side view of ODH-GLC: the protein is shown in light blue, the FAD cofactor in yellow, GLC in orange ( $\beta$ anomer) or gold ( $\alpha$ anomer); $\mathrm{O}$ atoms are in red, $\mathrm{N}$ atoms in blue. In both structures the $\beta$ anomer is closer to the FAD cofactor than the $\alpha$ anomer, and oriented with hydrogen bonds between the reactive $\mathrm{O} 1$ atom and 
His528 and the FAD N5 atom. Both sugars are sandwiched on top of Tyr64 by the aromatic residues Phe421, Phe416 and Trp430. Water molecules are depicted as red spheres. Hydrogen bonds (distance $<3.2 \AA$ ) are represented with dotted lines.

Fig. 7 External sugar-binding sites (SBSs) in ODH-LMB. All three sites are located on the convex face of ODH, whose surface is represented in the center and colored in green. LMB molecules are represented in sticks and colored in pink ( $\mathrm{C}$ atoms); $\mathrm{O}$ atoms are in red, $\mathrm{N}$ atoms in blue. The three sites are numbered according to their proximity to the active site entrance, located on the concave face of the protein (not shown). The three boxes show details of the interactions between protein and sugars. Water molecules are depicted as red spheres. Hydrogen bonds (distance <3.2 $\AA$ ) are represented with dotted lines. The $2 \mathrm{~F}_{\mathrm{o}}-\mathrm{F}_{\mathrm{c}}$ electron density map is also depicted around LMB molecules and contoured at $1 \sigma$.

Fig. 8 Schematic representation of the possible cleavage products of mixed $\beta(1 \rightarrow 3,1 \rightarrow 4)$ glucans, as found in hemicellulose. Depending on the type of cleavage performed by lytic enzymes, saccharides with different types of glycosidic bonds can be released. ODH is able to oxidize GLC, LMB and GTB (in green), but not active on CLB and GTA (in red). Some carbon atoms of glucosyl units in substrate and cleavage products are labelled. 
Figures

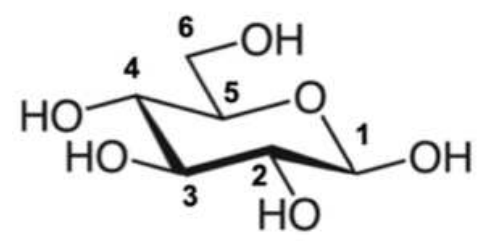

D-glucose

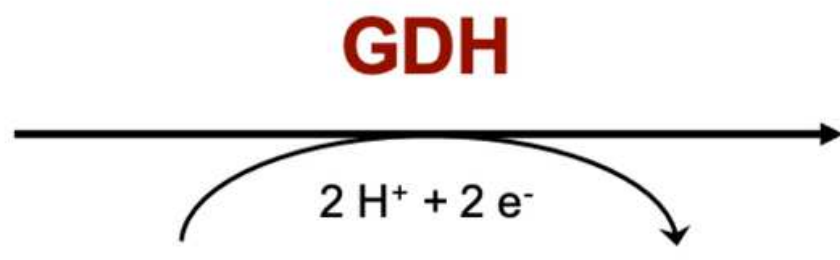

FAD

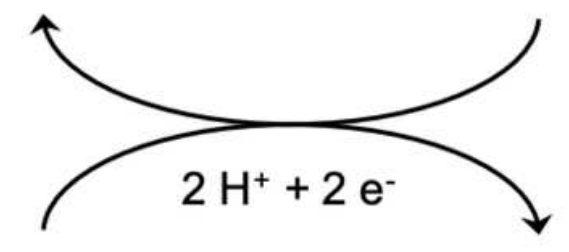

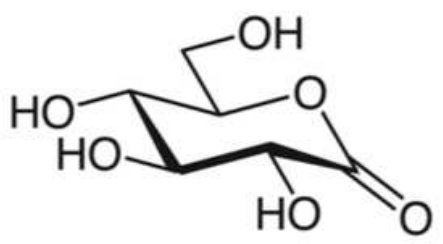

D-glucono- $\delta$-lactone
Quinones
FADU 2<smiles>Oc1ccc(O)cc1</smiles>

Hydroquinones

\section{Figure 1}

Catalytic cycle of GDHs. The enzyme in the oxidized resting state accepts electrons from a reducing sugar, like D-glucose, which is oxidized at the $\mathrm{C} 1$ position and converted into D-glucono- $\delta$-lactone; this causes the protein solution to turn from yellow to colorless, due to the reduction of the FAD cofactor. In the second half-reaction electrons are transferred from reduced FADH2 to aromatic electron acceptors, such as quinones.

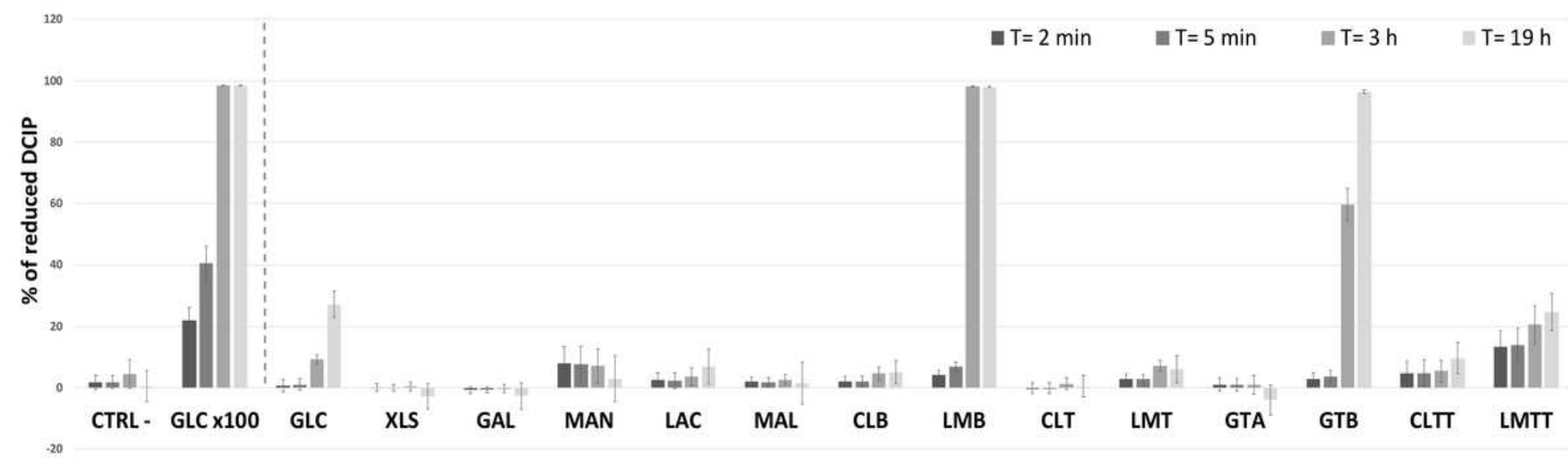

\section{Figure 2}

ODH substrate screening. Histograms correspond to the amount of DCIP which is reduced over time using a set of 14 sugars as electron donors for $\mathrm{ODH}$ reaction. Columns are colored using a grey scale 
code from black to light grey ( $t=2$ min to $t=19 \mathrm{~h}$, respectively). Sugar substrates were all tested at the same concentration (2.5 mM). Negative (CTRL -) and positive (GLCx100) control reactions were conducted with no substrate and with $250 \mathrm{mM} \mathrm{GLC}$ respectively and are reported on the left of the dashed line. Absorbance decrease at $520 \mathrm{~nm}$ was converted into percentage of reduced DCIP, using as $100 \%$ the absorbance of a solution containing no DCIP. Error bars represent the standard deviation of three independent experiments.
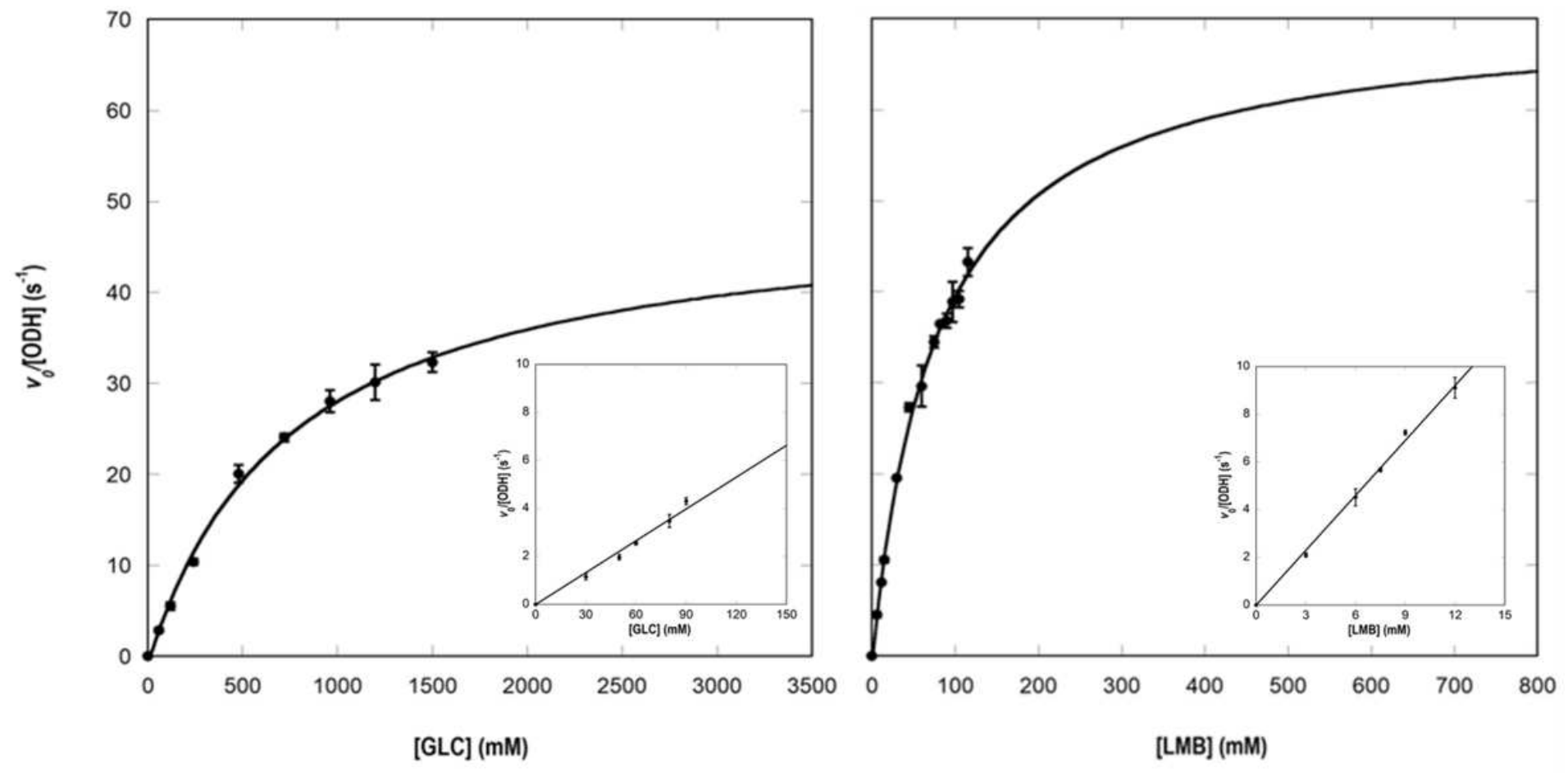

Figure 3

Initial rates of $\mathrm{ODH}$ reaction as a function of substrate concentration. $\mathrm{ODH}$ enzymatic kinetic assays were performed in the presence of GLC (left panel) and LMB (right panel) at different concentrations. Data were fitted to the standard Michaelis-Menten hyperbolic equation. Insets represent a linear plot of initial velocities of $\mathrm{ODH}$ at low substrate concentrations in the linear region of the Michaelis-Menten hyperbola. Error bars represent the standard deviation of three independent experiments. 


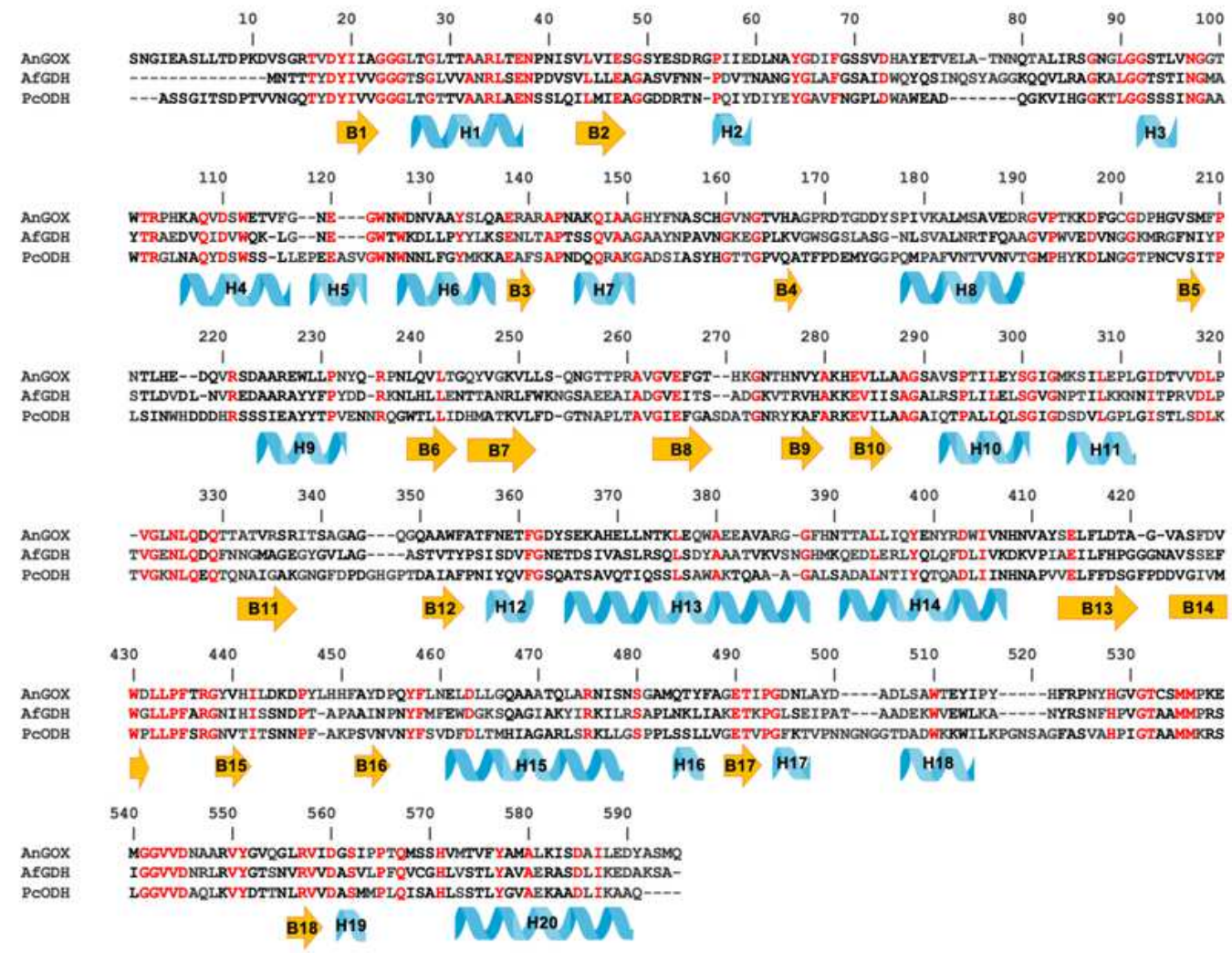
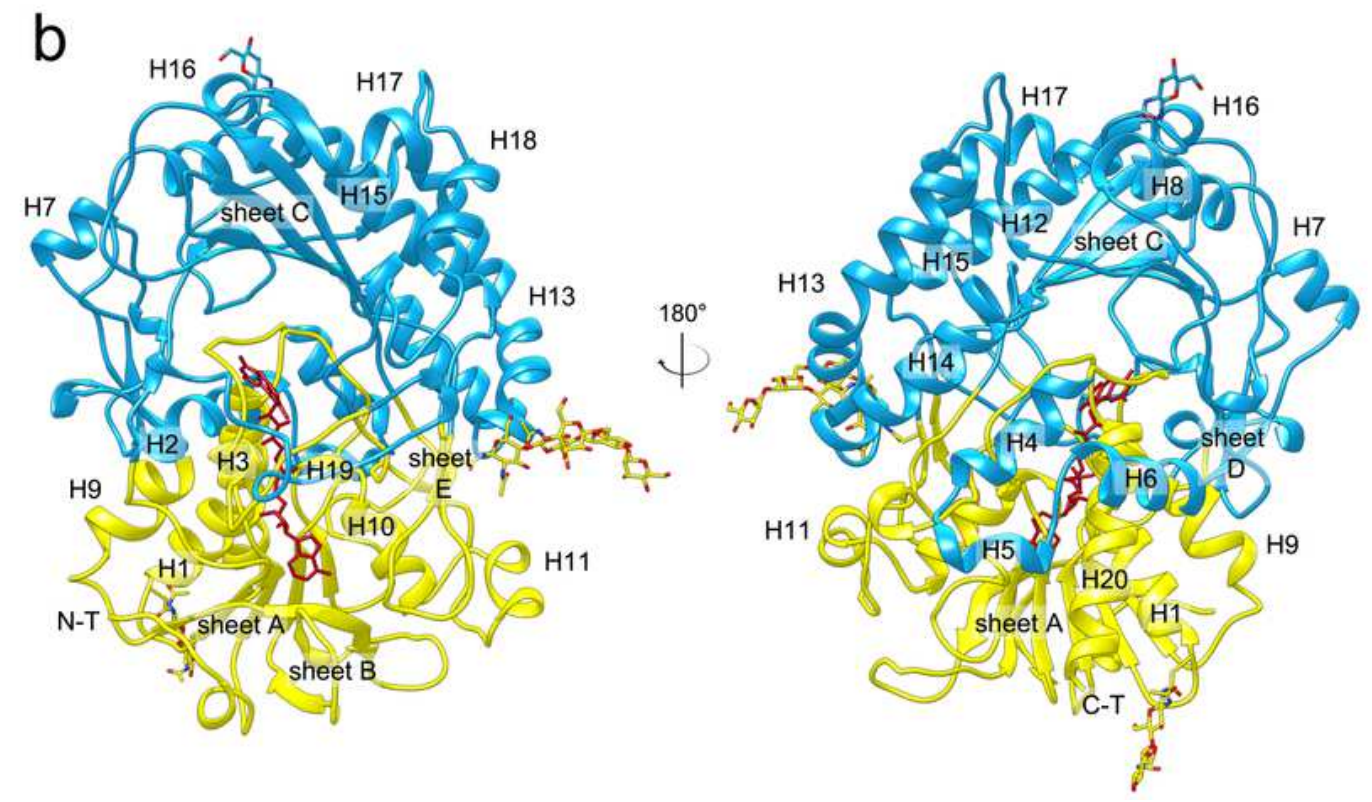

\section{Figure 4}

Overall structure of $\mathrm{ODH}$ and sequence alignment with homologous enzymes of known structure. a Sequence alignment of ODH, AfGDH and AnGOX based on structural superposition. Numbers refer to $\mathrm{ODH}$ sequence; conserved residues are colored in red. Secondary structure elements are assigned for $\mathrm{ODH}$ : $\beta$-strands are in orange and a-helices in light blue. b Ribbon representation of the whole threedimensional structure of ligand-free $\mathrm{ODH}$, with secondary structures assignment for $\beta$-sheets and $\mathrm{a}$ - 
helices $(H)$. The FAD-binding domain is shown in yellow, the substrate-binding domain in light blue, the FAD cofactor in dark red sticks. Glycosylations are represented in sticks, with $\mathrm{C}$ atoms in yellow or light blue, $\mathrm{O}$ in red and $\mathrm{N}$ in blue.
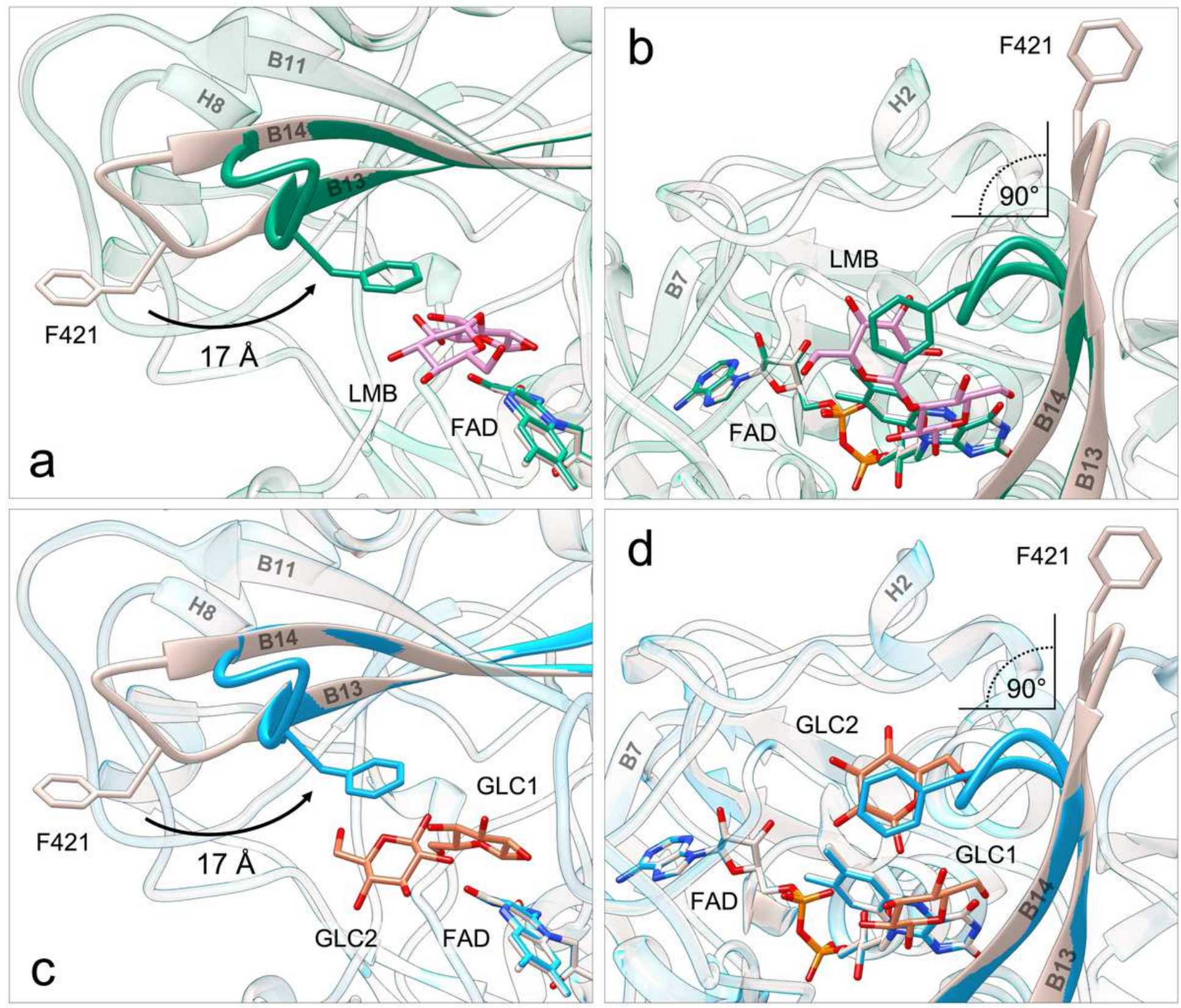

\section{Figure 5}

Conformational rearrangement of the substrate-binding loop upon sugar binding. Superposition of ligand-free ODH (gray) and ODH-LMB (green): a side view (perpendicular to the loop hinge axis), b top view (along the loop hinge axis). Superposition of ligand-free ODH (gray) and ODH-GLC (light blue) structures: $\mathrm{c}$ side view, $\mathrm{d}$ top view. LMB and GLC $\mathrm{C}$ atoms are in pink and orange respectively, $\mathrm{O}$ atoms in red and $\mathrm{N}$ atoms in blue. For clarity sake, only $\beta$ anomers are represented. Phe421 (also shown in sticks) is exposed to the bulk in the ligand free structure and shifts $\sim 17 \AA$ toward the active site upon binding of 
sugars, establishing $\mathrm{CH}-\pi$ interactions with the non-reducing glucosyl unit of LMB, or with GLC2 in ODHGLC. This movement causes the substrate-binding loop to rotate by $\sim 90^{\circ}$ and wrap toward the active site.
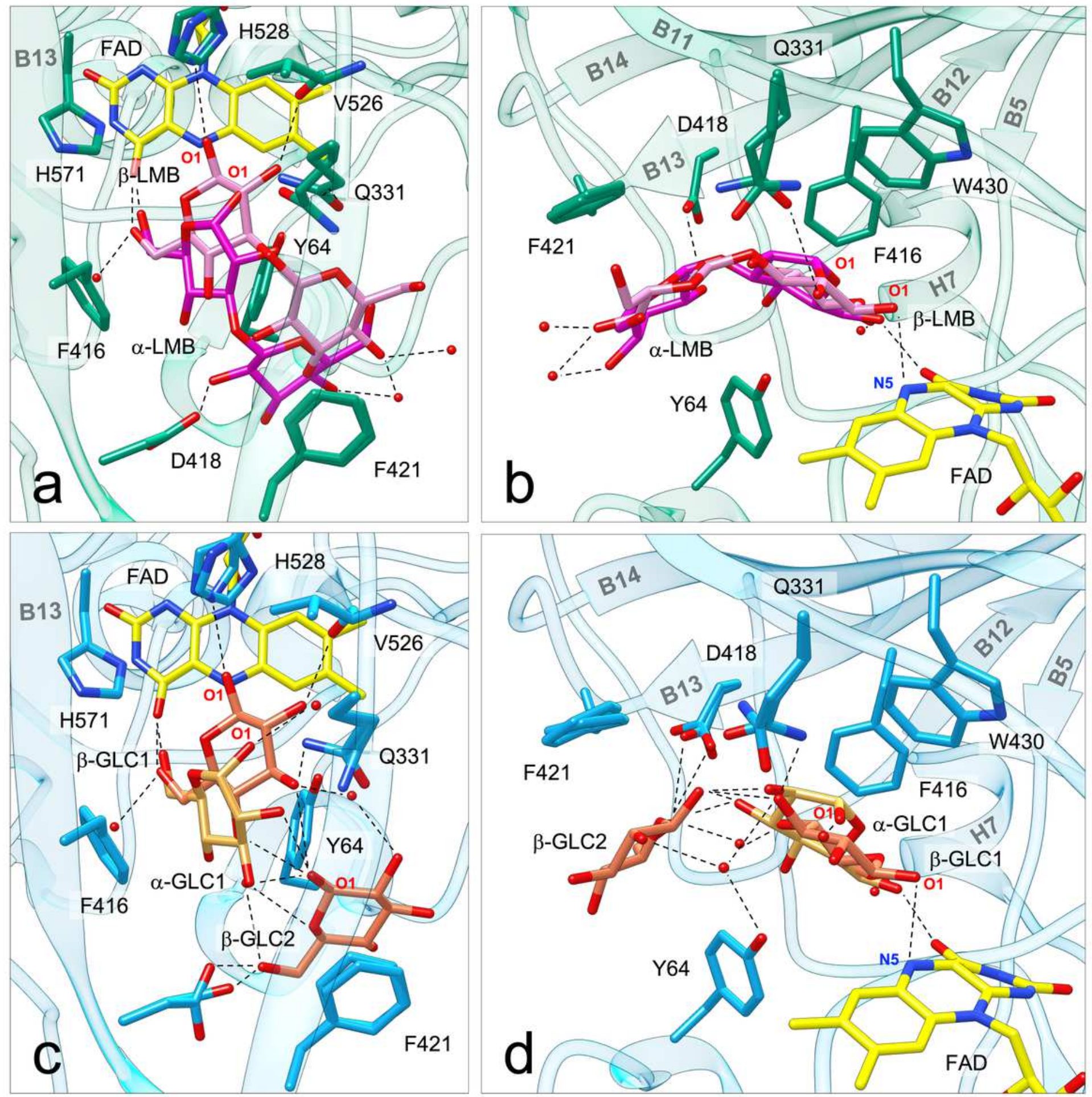

Figure 6

Sugar substrates bound to ODH active site. a Top view (perpendicular to the isoalloxazine plane) and $b$ side view of ODH-LMB: the protein is shown in green, the FAD cofactor in yellow, LMB in pink ( $\beta$ anomer) or magenta ( $a$ anomer). c Top view and d side view of ODH-GLC: the protein is shown in light blue, the 
FAD cofactor in yellow, GLC in orange ( $\beta$ anomer) or gold ( $a$ anomer); 0 atoms are in red, $\mathrm{N}$ atoms in blue. In both structures the $\beta$ anomer is closer to the FAD cofactor than the $\alpha$ anomer, and oriented with hydrogen bonds between the reactive 01 atom and His528 and the FAD N5 atom. Both sugars are sandwiched on top of Tyr64 by the aromatic residues Phe421, Phe416 and Trp430. Water molecules are depicted as red spheres. Hydrogen bonds (distance $<3.2 \AA$ ) are represented with dotted lines.

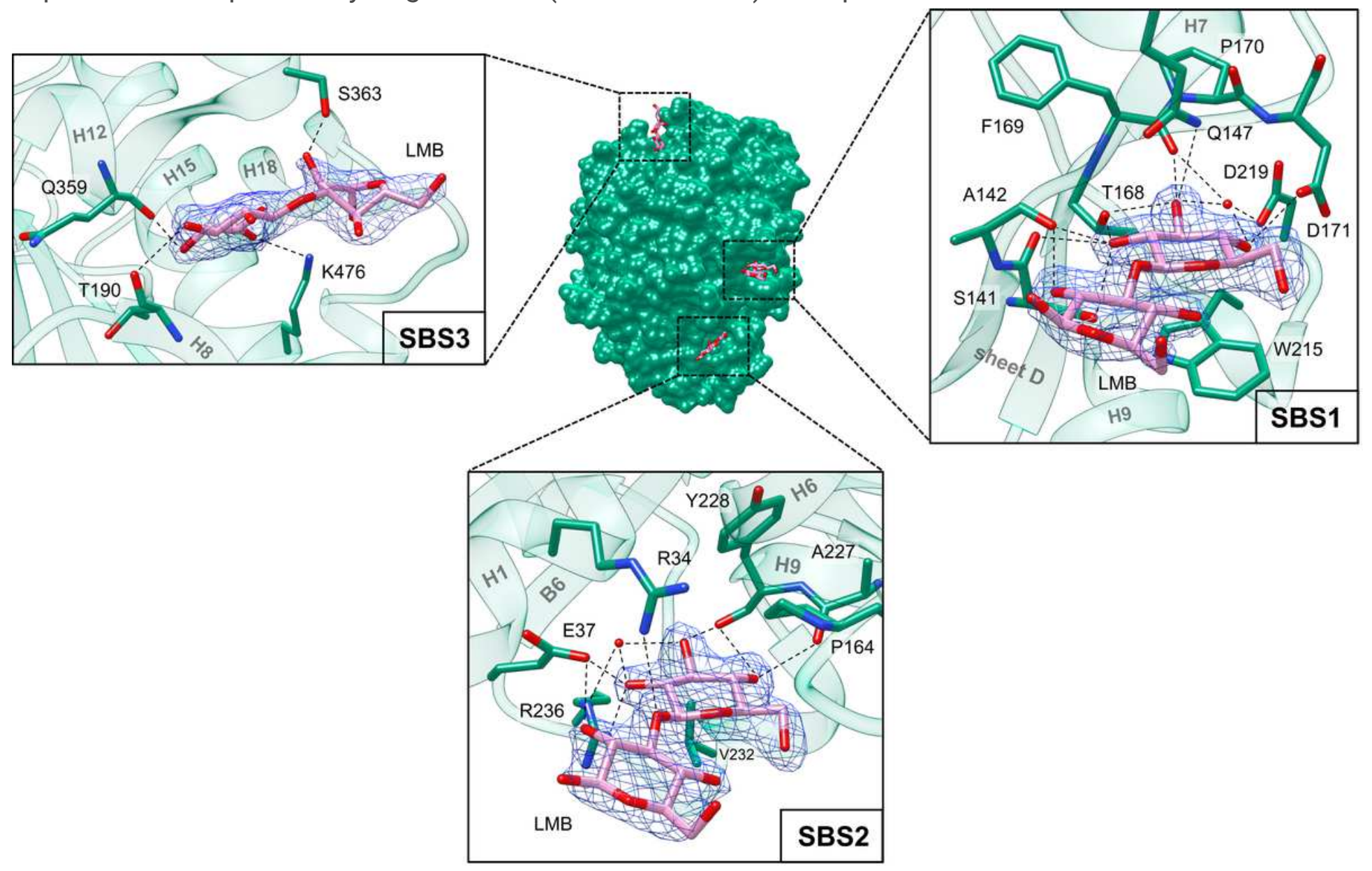

\section{Figure 7}

External sugar-binding sites (SBSs) in ODH-LMB. All three sites are located on the convex face of ODH, whose surface is represented in the center and colored in green. LMB molecules are represented in sticks and colored in pink ( $\mathrm{C}$ atoms); $\mathrm{O}$ atoms are in red, $\mathrm{N}$ atoms in blue. The three sites are numbered according to their proximity to the active site entrance, located on the concave face of the protein (not shown). The three boxes show details of the interactions between protein and sugars. Water molecules are depicted as red spheres. Hydrogen bonds (distance $<3.2 \AA$ ) are represented with dotted lines. The 2FoFc electron density map is also depicted around LMB molecules and contoured at $\sigma$. 


\section{Mixed $\beta(1 \rightarrow 3,1 \rightarrow 4)$ glucans}

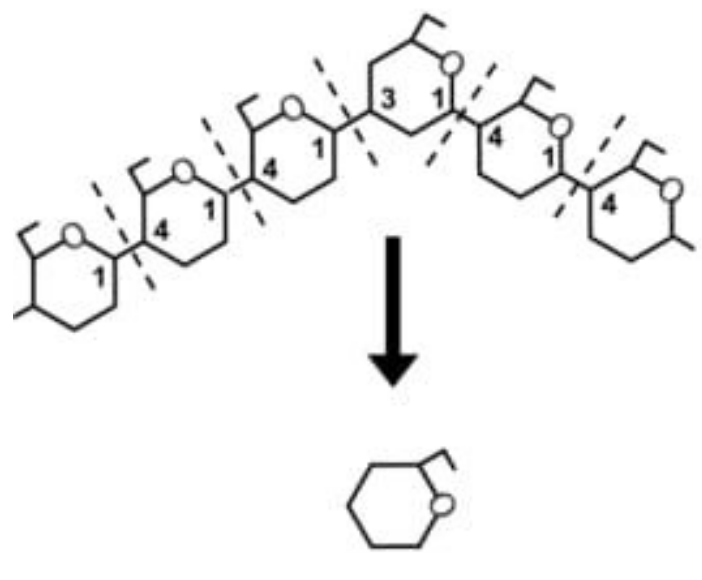

GLC
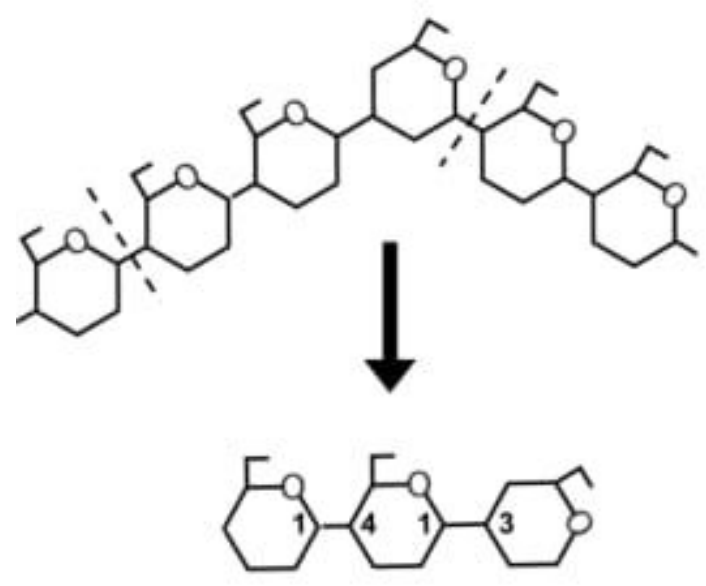

GTB
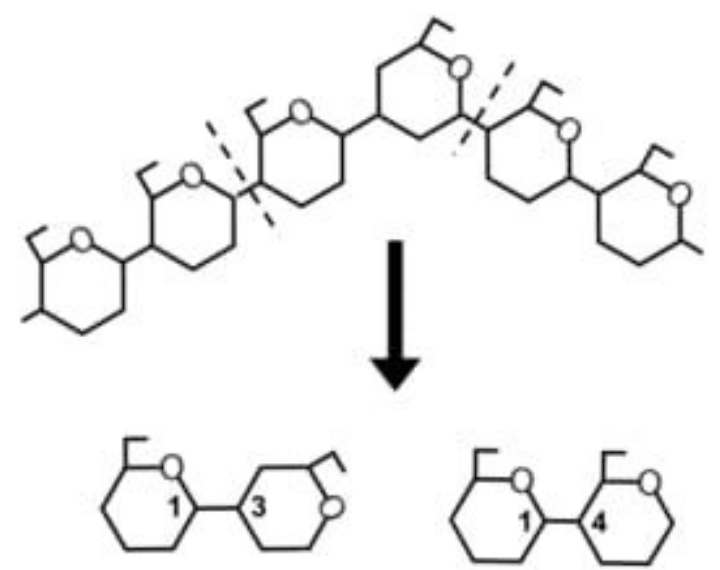

LMB

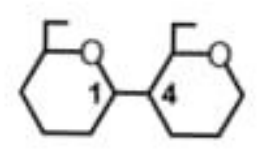

CLB
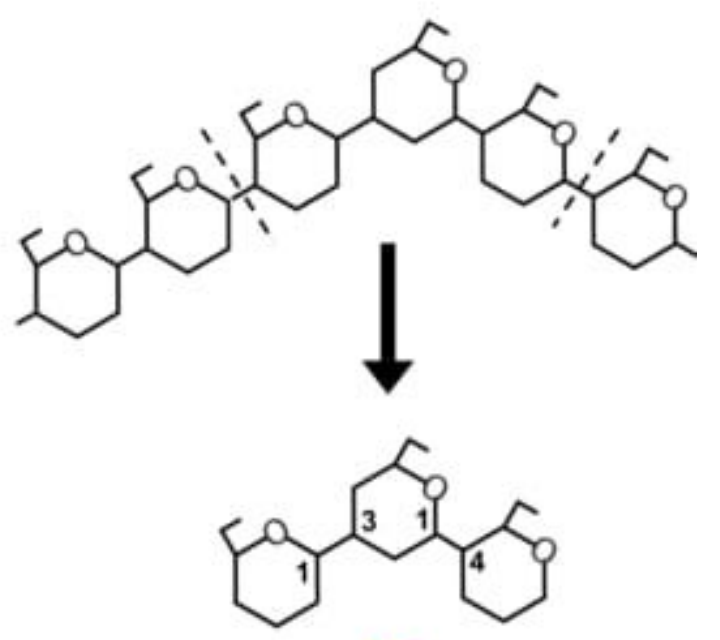

GTA

Figure 8

Schematic representation of the possible cleavage products of mixed $\beta(1 \otimes 3,1 \otimes 4)$ glucans, as found in hemicellulose. Depending on the type of cleavage performed by lytic enzymes, saccharides with different types of glycosidic bonds can be released. ODH is able to oxidize GLC, LMB and GTB (in green), but not active on CLB and GTA (in red). Some carbon atoms of glucosyl units in substrate and cleavage products are labelled.

\section{Supplementary Files}

This is a list of supplementary files associated with this preprint. Click to download.

- AdditionalFile1.pdf 
- AdditionalFile2.pdf 